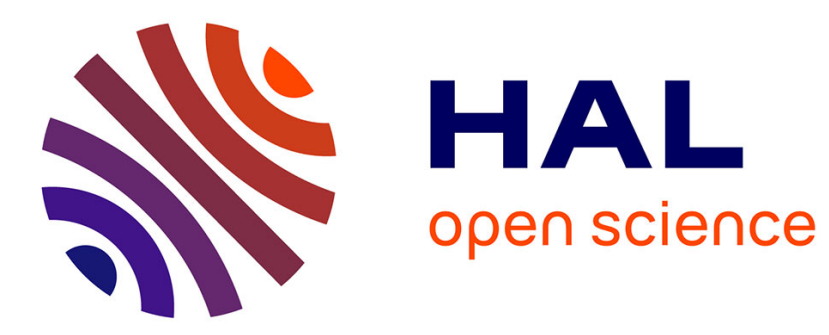

\title{
Resistance to antibiotics: limit theorems for a stochastic SIS model structured by level of resistance
}

Pierre-Yves Boëlle, Guy Thomas

\section{To cite this version:}

Pierre-Yves Boëlle, Guy Thomas. Resistance to antibiotics: limit theorems for a stochastic SIS model structured by level of resistance. Journal of Mathematical Biology, 2016, 10.1007/s00285-016-0996-2 . hal-01303276

\section{HAL Id: hal-01303276 \\ https://hal.sorbonne-universite.fr/hal-01303276}

Submitted on 17 Apr 2016

HAL is a multi-disciplinary open access archive for the deposit and dissemination of scientific research documents, whether they are published or not. The documents may come from teaching and research institutions in France or abroad, or from public or private research centers.
L'archive ouverte pluridisciplinaire HAL, est destinée au dépôt et à la diffusion de documents scientifiques de niveau recherche, publiés ou non, émanant des établissements d'enseignement et de recherche français ou étrangers, des laboratoires publics ou privés. 


\title{
Resistance to antibiotics: limit theorems for a stochastic SIS model structured by level of resistance
}

\author{
Pierre-Yves Boëlle and Guy Thomas \\ Sorbonne Universités, UPMC Univ Paris 06, INSERM, Institut \\ Pierre Louis d'épidémiologie et de Santé Publique (IPLESP UMRS \\ 1136), F75012, Paris, France
}

\begin{abstract}
The rise of bacterial resistance to antibiotics is a major Public Health concern. It is the result of two interacting processes: the selection of resistant bacterial strains under exposure to antibiotics and the dissemination of bacterial strains throughout the population by contact between colonized and uncolonized individuals. To investigate the resulting time evolution of bacterial resistance, Temime et al (2003) developed a stochastic SIS model, which was structured by the level of resistance of bacterial strains. Here we study the asymptotic properties of this model when the population size is large. To this end, we cast the model within the framework of measure valued processes, using point measures to represent the pattern of bacterial resistance in the compartments of colonized individuals. We first show that the suitably normalized model tends in probability to the solution of a deterministic differential system. Then we prove that the process of fluctuations around this limit tends in law to a Gaussian process in a space of distributions. These results, which generalize those of Kurtz (1981, chap. 8) on SIR models, support the validity of the deterministic approximation and quantify the rate of convergence.
\end{abstract}

\section{Introduction}

During the last decades, bacterial resistance to antibiotics has become a major Public Health concern (Cars et al, 2008). The case of S. Pneumoniae, a pathogen responsible for respiratory infections, otitis, and meningitis, is particularly illustrative of the phenomenon, with prevalences of intermediate or resistant pneumococcal strains exceeding $10 \%$ in $50 \%$, and $25 \%$ in 15\%, of European countries (EARS-Network, 2012). The selection of resistant strains is the result of several interacting processes. At the bacterial level, point mutations and horizontal transfers of genetic material lead to changes in antibiotic resistance 
with a large range of effects (Martinez, 2008). In the absence of exposure to antibiotics, it is thought that most mutated strains do not give birth to significant populations, since they are rapidly outgrown by competing antibiotic-sensitive strains because of differences in fitness (Maher et al, 2012). In the presence of antibiotics however, antibiotic-sensitive strains are selectively eliminated, so that antibiotic-resistant strains will develop and colonize the available niche. Exposure to antibiotics in human or animal populations is widespread, occuring through prescribed or over-the-counter medical treatments, and other routes such as food consumption. Once an antibiotic-resistant strain has been selected in a host, it may disseminate across the population through inter-individual contacts.

Temime et al (2003) developed a model which integrates the processes operating both at the bacterial and the human population levels, together with their interactions. The model was cast in the form of an SIS stochastic model whose compartments specify the status of individuals with respect to colonization by bacteria, and exposure to antibiotics. We use the term 'colonization' rather than 'infection', since asymptomatic carriage is frequent. Each colonized individual was further described by the level of resistance of the colonizing bacterial strain which, in the case of penicillins, spreads over a continuum of values. Because of this extra structure, the model belongs to the class of individual-based models, and its properties in the large population limit cannot be investigated following the approach of Kurtz (1981), as e.g. in Andersson and Britton (2000, chap.5). Although a law of large numbers was suggested by heuristic reasoning (Temime et al 2005), no rigorously established result is yet available.

In this paper, we consider the SIS model of Temime et al (2003), and represent the resistance patterns of the colonized individuals by point measures. This formalism allows a thorough investigation of the large population limit using the approach of Fournier and Méléard (2004) and Méléard (1998). A similar line of attack was used in Clémençon et al (2008) to study an SIR epidemic model with an age structure. More specifically, we prove both a law of large numbers and a central limit theorem for the suitably normalized model as the population size tends to infinity.

\section{Model and notations}

The population is assumed to be of constant size $n$, and is partitioned into five compartments as depicted in Figure 1 (see Temime et al (2003) for details): 4 compartments characterize the status of individuals with respect to both colonization by $S$. Pneumoniae and exposure to antibiotics; the fifth compartment corresponds to the so-called refractory phase, during which individuals cannot be colonized due to acquired immunity with respect to the bacterial agent: such a phase follows spontaneous decolonization but is not observed when decolonization results from exposure to antibiotics (Prellner et al, 1999). Uncolonized unexposed individuals are in compartment $X_{u}$; individuals in refractory phase following spontaneous decolonization are in compartment $X_{r}$; uncolonized ex- 
posed individuals are in compartment $X_{e}$; colonized unexposed individuals are in compartment $Y_{u}$; and colonized exposed individuals are in compartment $Y_{e}$. Colonized individuals are further characterized by their level of resistance to antibiotics, modeled as a continuous positive variable. Uncolonized individuals may become colonized through contact with colonized individuals. Colonized individuals may undergo decolonization, either spontaneously or through exposure to antibiotics. If exposed, they may in addition undergo an increase of their resistance level by selection of a population of genetically altered bacterial strains.

Let $\delta_{m}$ denote the Dirac measure at $m$, and $\mathcal{M}=\left\{\sum_{i=1}^{k} \delta_{m_{i}}, m_{i}>0, k \leqslant n\right\}$ denote the set of point measures on $\mathbb{R}_{+}=(0, \infty)$, with total mass bounded by $n$. For $\nu=\sum_{i=1}^{k} \delta_{m_{i}} \in \mathcal{M}$ and $f$ a measurable bounded function on $\mathbb{R}_{+}$, we set $\langle\nu, f\rangle=\int_{\mathbb{R}_{+}} \nu(d m) f(m)=\sum_{i=1}^{k} f\left(m_{i}\right)$.

For all time $t$, the state of the population is described by the vector $Z(t)=$ $\left(Z_{X_{u}}(t), Z_{X_{r}}(t), Z_{X_{e}}(t), Z_{Y_{u}}(t), Z_{Y_{e}}(t)\right)$, which takes values in the state space $E=\{0,1, \ldots, n\}^{3} \times \mathcal{M}^{2}$. More specifically,

- $Z_{X_{u}}(t)$ is the number of uncolonized unexposed individuals,

- $Z_{X_{r}}(t)$ is the number of individuals in refractory phase,

- $Z_{X_{e}}(t)$ is the number of uncolonized exposed individuals,

- $Z_{Y_{u}}(t)$ is the random measure $\sum_{i=1}^{N_{Y_{u}}(t)} \delta_{a_{i}(t)}$, where $N_{Y_{u}}(t)=\left\langle Z_{Y_{u}}(t), 1\right\rangle$ is the number of colonized unexposed individuals, and $a_{1}(t), \ldots, a_{N_{Y_{u}}(t)}(t)$ denote their respective resistance levels,

- $Z_{Y_{e}}(t)$ is the random measure $\sum_{j=1}^{N_{Y_{e}}(t)} \delta_{b_{j}(t)}$, where $N_{Y_{e}}(t)=\left\langle Z_{Y_{e}}(t), 1\right\rangle$ is the number of colonized exposed individuals and $b_{1}(t), \ldots, b_{N_{Y_{e}}(t)}(t)$ denote their respective resistance levels.

The dynamics of the population are driven by the following transitions:

- An unexposed individual, colonized or uncolonized, may become exposed, at rate $\alpha_{o n}$.

- An exposed individual, colonized or uncolonized, may become unexposed, at rate $\alpha_{\text {end }}$.

The rates $\alpha_{\text {on }}$ and $\alpha_{\text {end }}$ do not differ between colonized and uncolonized individuals, since the majority of $S$. Pneumoniae carriers are asymptomatic, and exposure to antibiotics thus mainly results from prescription for unrelated infectious diseases.

- A colonized unexposed individual may undergo spontaneous decolonization and enter the refractory phase, at rate $\lambda_{u}$.

- An individual in the refractory phase may leave this state and enter the uncolonized unexposed compartment, at rate $\alpha_{r}$. 


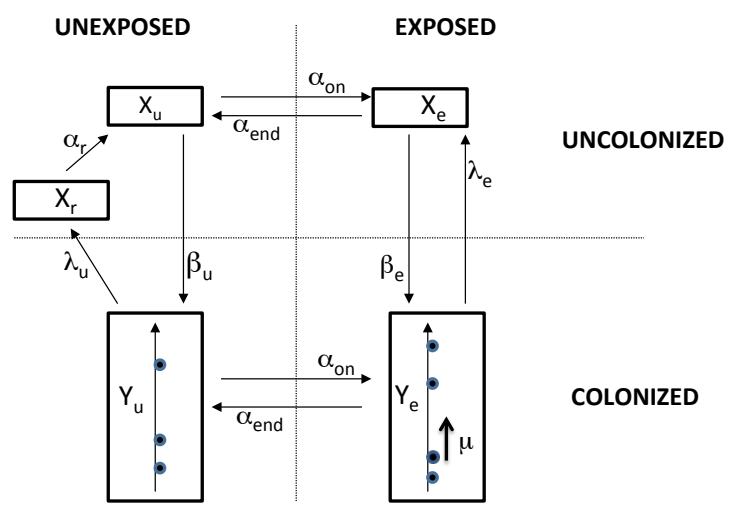

Figure 1: Schematic description of the compartmental model, with the respective transition rates. The vertical axes in the Colonized compartments represent the scale of resistance levels, and the dots represent Dirac masses at the resistance levels of the colonized individuals. 
- A colonized exposed individual, with resistance level $b$, may undergo decolonization, at rate $\lambda_{e}(b)$. The rate of decolonization under exposure to antibiotics is the sum of the spontaneous rate $\lambda_{u}$ and the rate due to treatment effect. Since the latter rate decreases as the resistance level of the colonizing strain increases, $\lambda_{e}$ is a non increasing function on $\mathbb{R}_{+}$, tending to $\lambda_{u}$ at infinity. We let $\bar{\lambda}_{e}=\lambda_{e}(0)$.

- An uncolonized unexposed individual may get colonized through contact with a given colonized individual, exposed or unexposed, at rate $\beta_{u} / n$. The newly colonized individual acquires the resistance level of his contaminating contact.

- An exposed uncolonized individual may get colonized through contact with a given colonized individual, exposed or unexposed, with resistance level $m$, at rate $\beta_{e}(m) / n$. To reflect the fact that the rate of colonization by contact under exposure to antibiotics is higher when the resistance level of the colonizing strain is higher, $\beta_{e}$ is a nonnegative nondecreasing function on $\mathbb{R}_{+}$, assumed bounded from above by $\bar{\beta}_{e}$. The resistance level of the newly colonized individual is $m$.

The normalizations $\beta_{u} / n$ and $\beta_{e} / n$ are standard in the formulation of epidemic models (Andersson and Britton, 2000, section 2.1). They correspond to the fact that in a mixing population of size $n$, each individual has probability $1 /(n-1) \simeq 1 / n$ of making contact with another given individual.

In Temime et al (2003), $\beta_{e}(b)$ ranges from 0 to $2 \beta_{u}$ as $b$ ranges from 0 to infinity. This reflects the fact that: if the transmitted strain is not resistant to antibiotics, treatment hinders colonization of the antibiotic exposed individual; whereas if it is highly resistant, treatment facilitates colonization as it clears the endogenous bacterial flora.

- A colonized exposed individual may undergo a genetic event, at rate $\mu$. As a result, the individual's resistance level increases by an amount of $h$, where $h$ is a positive random variable with distribution $R(d h)=\rho(h) d h$. While genetic events may lead to drops in resistance level, such events are not observed, since, under exposure to antibiotics, bacterial strains with lower resistance will not be able to establish as the dominant strain.

In the sequel, the components of a generic process $U$ with values in $E$ will be denoted by $U_{X_{u}}, U_{X_{r}}, U_{X_{e}}, U_{Y_{u}}$, and $U_{Y_{e}}$, respectively. For $f, g: \mathbb{R}_{+} \rightarrow \mathbb{R}$ measurable and bounded, we define

$$
U^{f, g}=\left(U_{X_{u}}, U_{X_{r}}, U_{X_{e}}, U_{Y_{u}}^{f}, U_{Y_{e}}^{g}\right),
$$

where $U_{Y_{u}}^{f}=\left\langle U_{Y_{u}}, f\right\rangle$ and $U_{Y_{e}}^{g}=\left\langle U_{Y_{e}}, g\right\rangle$.

For $f: \mathbb{R} \rightarrow \mathbb{R}$, and $h \geqslant 0$, we let $\tau_{h} f(\cdot)=f(\cdot+h)$.

The notation $\mathbb{1}_{A}$ stands for the indicator function of the set $A$.

Throughout the paper, $K$ will denote a generic constant, whose meaning may change from line to line. 


\subsection{Poisson measure representation}

The process $(Z(t))_{t \geqslant 0}$ will be realized as the solution of a system of stochastic differential equations driven by Poisson measures. On a probability space $(\Omega, \mathcal{F}, \mathbb{P})$ we consider the following independent random elements:

1. The initial state $Z(0) \in E$, which is specified through

- The vector $\left(Z_{X_{u}}(0), Z_{X_{r}}(0), Z_{X_{e}}(0), N_{Y_{u}}(0), N_{Y_{e}}(0)\right)$, giving the initial numbers of individuals in the compartments, assumed to be multinomial with index $n$, and probabilities $\left(\theta_{X_{u}}, \theta_{X_{r}}, \theta_{X_{e}}, \theta_{Y_{u}}, \theta_{Y_{e}}\right)$.

- The initial point measures $Z_{Y_{u}}(0)=\sum_{i=1}^{N_{Y_{u}}(0)} \delta_{a_{i}}$ and $Z_{Y_{e}}(0)=$ $\sum_{j=1}^{N_{Y_{e}}(0)} \delta_{b_{j}}$, where $\left(a_{i}\right)_{i \geqslant 1}$, and $\left(b_{j}\right)_{j \geqslant 1}$ are two sequences of i.i.d. positive random variables with respective distributions $P_{u}$ and $P_{e}$.

2. The following Poisson measures:

- exposure onset Poisson measures $Q_{X_{u}}^{o n}(d s, d i)$ and $Q_{Y_{u}}^{o n}(d s, d i)$, with common intensity measure $\alpha_{o n} d s \otimes d i$,

- exposure ending Poisson measures $Q_{X_{e}}^{e n d}(d s, d i)$ and $Q_{Y_{e}}^{e n d}(d s, d i)$, with common intensity measure $\alpha_{e n d} d s \otimes d i$,

- decolonization Poisson measures $Q_{Y_{u}}^{\text {decol }}(d s, d i)$ and $Q_{Y_{e}}^{\text {decol }}(d s, d i, d v)$, with respective intensity measures $\lambda_{u} d s \otimes d i$ and $\bar{\lambda}_{e} d s \otimes d i \otimes d v$,

- colonization Poisson measures $Q_{X_{u}}^{c o l}(d s, d i, d j)$ and $Q_{X_{e}}^{c o l}(d s, d i, d j, d v)$, with respective intensity measures $\frac{\beta_{u}}{n} d s \otimes d i \otimes d j$ and $\frac{\bar{\beta}_{e}}{n} d s \otimes d i \otimes$ $d j \otimes d v$,

- a refractory phase exit Poisson measure $Q_{X_{r}}^{\text {exit }}(d s, d i)$, with intensity measure $\alpha_{r} d s \otimes d i$

- a mutation Poisson measure $Q_{Y_{e}}^{m u t}(d s, d i, d h)$, with intensity measure $\mu d s \otimes d i \otimes \rho(h) d h$,

where $d s$ and $d h$ are Lebesgue measure on $\mathbb{R}^{+}, d i$ and $d j$ are counting measures on $\mathbb{N}^{*}$, and $d v$ is Lebesgue measure on $[0,1]$. We let $\left(\mathcal{F}_{t}\right)_{t \geqslant 0}$ denote the canonical filtration generated by $Z(0)$ and the above Poisson measures.

The model $(Z(t))_{t \geqslant 0}$ is defined as the $\left(\mathcal{F}_{t}\right)$-Markov process, which is the unique solution of the system of stochastic differential equations

$$
\left\{\begin{array}{l}
Z_{X_{u}}(t)=Z_{X_{u}}(0)-I_{X_{u}}^{o n}(t)+I_{X_{e}}^{e n d}(t)+I_{X_{r}}^{e x i t}(t)-\left\langle I_{X_{u}}^{c o l}(t), 1\right\rangle \\
Z_{X_{r}}(t)=Z_{X_{r}}(0)+\left\langle I_{Y_{u}}^{\text {decol }}(t), 1\right\rangle-I_{X_{r}}^{e x i t}(t) \\
Z_{X_{e}}(t)=Z_{X_{e}}(0)+I_{X_{u}}^{o n}(t)-I_{X_{e}}^{e n d}(t)+\left\langle I_{Y_{e}}^{\text {decol }}(t), 1\right\rangle-\left\langle I_{X_{e}}^{c o l}(t), 1\right\rangle \\
Z_{Y_{u}}(t)=Z_{Y_{u}}(0)-I_{Y_{u}}^{o n}(t)+I_{Y_{e}}^{e n d}(t)-I_{Y_{u}}^{\text {decol }}(t)+I_{X_{u}}^{c o l}(t) \\
Z_{Y_{e}}(t)=Z_{Y_{e}}(0)+I_{Y_{u}}^{o n}(t)-I_{Y_{e}}^{e n d}(t)-I_{Y_{e}}^{\text {decol }}(t)+I_{X_{e}}^{c o l}(t)+I_{Y_{e}}^{m u t}(t)
\end{array}\right.
$$

where

$$
I_{X_{u}}^{o n}(t)=\int_{0}^{t} \int_{\mathbb{N}^{*}} \mathbb{1}_{\left\{i \leqslant Z_{X_{u}}\left(s_{-}\right)\right\}} Q_{X_{u}}^{\text {on }}(d s, d i)
$$




$$
\begin{aligned}
& I_{Y_{u}}^{o n}(t)=\int_{0}^{t} \int_{\mathbb{N}^{*}} \mathbb{1}_{\left\{i \leqslant N_{Y_{u}}\left(s_{-}\right)\right\}} \delta_{a_{i}\left(s_{-}\right)} Q_{Y_{u}}^{o n}(d s, d i) \\
& I_{X_{e}}^{\text {end }}(t)=\int_{0}^{t} \int_{\mathbb{N}^{*}} \mathbb{1}_{\left\{i \leqslant Z_{X_{e}}\left(s_{-}\right)\right\}} Q_{X_{e}}^{\text {end }}(d s, d i) \\
& I_{Y_{e}}^{\text {end }}(t)=\int_{0}^{t} \int_{\mathbb{N} *} \mathbb{1}_{\left\{i \leqslant N_{Y_{e}}\left(s_{-}\right)\right\}} \delta_{b_{i}\left(s_{-}\right)} Q_{Y_{e}}^{\text {end }}(d s, d i) \\
& I_{X_{r}}^{\text {exit }}(t)=\int_{0}^{t} \int_{\mathbb{N}^{*}} \mathbb{1}_{\left\{i \leqslant Z_{X_{r}}\left(s_{-}\right)\right\}} Q_{X_{r}}^{\text {exit }}(d s, d i) \\
& I_{X_{u}}^{c o l}(t)=\int_{0}^{t} \int_{\mathbb{N}^{*}} \int_{\mathbb{N}^{*}} \mathbb{1}_{\left\{i \leqslant Z_{X_{u}}\left(s_{-}\right)\right\}}\left(\mathbb{1}_{\left\{j \leqslant N_{Y_{u}}\left(s_{-}\right)\right\}} \delta_{a_{j}\left(s_{-}\right)}\right. \\
& \left.+\mathbb{1}_{\left\{1 \leqslant j-N_{Y_{u}}\left(s_{-}\right) \leqslant N_{Y_{e}}\left(s_{-}\right)\right\}} \delta_{b_{j-N_{Y_{u}}\left(s_{-}\right)}\left(s_{-}\right)}\right) Q_{X_{u}}^{c o l}(d s, d i, d j) \\
& I_{X_{e}}^{c o l}(t)=\int_{0}^{t} \int_{\mathbb{N}^{*}} \int_{\mathbb{N}^{*}} \int_{0}^{1} \mathbb{1}_{\left\{i \leqslant Z_{X_{e}}\left(s_{-}\right)\right\}}\left(\mathbb{1}_{\left\{j \leqslant N_{Y_{u}}\left(s_{-}\right)\right\}} \mathbb{1}_{\left\{v \leqslant \beta_{e}\left(a_{j}(s-)\right) / \bar{\beta}_{e}\right\}} \delta_{a_{j}\left(s_{-}\right)}\right. \\
& +\mathbb{1}_{\left\{1 \leqslant j-N_{Y_{u}}\left(s_{-}\right) \leqslant N_{Y_{e}}\left(s_{-}\right)\right\}} \mathbb{1}_{\left\{v \leqslant \beta_{e}\left(b_{j-N_{Y_{u}}\left(s_{-}\right)}(s-)\right) / \bar{\beta}_{e}\right\}} \\
& \left.\times \delta_{b_{j-N_{Y_{u}}\left(s_{-}\right)}\left(s_{-}\right)}\right) Q_{X_{e}}^{c o l}(d s, d i, d j, d v) \\
& I_{Y_{u}}^{\text {decol }}(t)=\int_{0}^{t} \int_{\mathbb{N}^{*}} \mathbb{1}_{\left\{i \leqslant N_{Y_{u}}\left(s_{-}\right)\right\}} \delta_{a_{i}\left(s_{-}\right)} Q_{Y_{u}}^{\text {decol }}(d s, d i) \\
& I_{Y_{e}}^{\text {decol }}(t)=\int_{0}^{t} \int_{\mathbb{N}^{*}} \int_{0}^{1} \mathbb{1}_{\left\{i \leqslant N_{Y_{e}}\left(s_{-}\right)\right\}} \mathbb{1}_{\left\{v \leqslant \lambda_{e}\left(b_{i}(s-)\right) / \bar{\lambda}_{e}\right\}} \delta_{b_{i}\left(s_{-}\right)} Q_{Y_{e}}^{\text {decol }}(d s, d i, d v) \\
& I_{Y_{e}}^{m u t}(t)=\int_{0}^{t} \int_{\mathbb{N}^{*}} \int_{0}^{1} \int_{\mathbb{R}_{+}} \mathbb{1}_{\left\{i \leqslant N_{Y_{e}}\left(s_{-}\right)\right\}}\left(\delta_{b_{i}\left(s_{-}\right)+h}-\delta_{b_{i}\left(s_{-}\right)}\right) Q_{Y_{e}}^{m u t}(d s, d i, d h) .
\end{aligned}
$$

Note that the above representation of $(Z(t))_{t \geqslant 0}$ yields a straightforward algorithm for pathwise simulation of the process.

\section{Law of large numbers}

To establish a law of large numbers, as $n \rightarrow \infty$, we consider the normalized process $Z^{n}=\frac{1}{n} Z$, with state space $E_{1}=[0,1]^{3} \times\left(\mathcal{M}_{1}\right)^{2}$, where $\mathcal{M}_{1}$ is the space of subprobability measures on $\mathbb{R}_{+}$. On $E_{1}$ we put the product topology induced by the usual topology on $[0,1]$, and the topology of weak convergence on $\mathcal{M}_{1}$.

If $S$ is a metric space, and $T>0, C([0, T], S)$ denotes the space of continuous functions from $[0, T]$ to $S$, equipped with the topology of uniform convergence, and $D([0, T], S)$ denotes the space of càdlàg functions from $[0, T]$ to $S$, equipped with the Skorohod topology (see e.g. Jacod and Shiryaev (2003, Chaper VI)).

To keep notations as concise as possible, given $f, g: \mathbb{R}_{+} \rightarrow \mathbb{R}$ measurable and bounded, we define two mappings, $\Psi^{f, g}$ and $\hat{\Psi}^{f, g}$.

The map $\Psi^{f, g}=\left(\Psi_{X_{u}}, \Psi_{X_{r}}, \Psi_{X_{e}}, \Psi_{Y_{u}}^{f}, \Psi_{Y_{e}}^{g}\right)$, is defined from $D\left([0, T], E_{1}\right)$ 
to $D\left([0, T], \mathbb{R}^{5}\right)$ as

$$
\begin{aligned}
& \Psi_{X_{u}}(U)(t)=\int_{0}^{t} d s\left(-\alpha_{o n} U_{X_{u}}(s)+\alpha_{r} U_{X_{r}}(s)+\alpha_{e n d} U_{X_{e}}(s)\right. \\
& \left.-\beta_{u} U_{X_{u}}(s)\left\langle U_{Y_{u}}(s)+U_{Y_{e}}(s), 1\right\rangle\right) \\
& \Psi_{X_{r}}(U)(t)=\int_{0}^{t} d s\left(-\alpha_{r} U_{X_{r}}(s)+\lambda_{u}\left\langle U_{Y_{u}}(s), 1\right\rangle\right) \\
& \Psi_{X_{e}}(U)(t)=\int_{0}^{t} d s\left(\alpha_{o n} U_{X_{u}}(s)-\alpha_{e n d} U_{X_{e}}(s)+\left\langle U_{Y_{e}}(s), \lambda_{e}\right\rangle\right. \\
& \left.-U_{X_{e}}(s)\left\langle U_{Y_{u}}(s)+U_{Y_{e}}(s), \beta_{e}\right\rangle\right) \\
& \Psi_{Y_{u}}^{f}(U)(t)=\int_{0}^{t} d s\left(-\left(\alpha_{o n}+\lambda_{u}\right)\left\langle U_{Y_{u}}(s), f\right\rangle+\alpha_{e n d}\left\langle U_{Y_{e}}(s), f\right\rangle\right. \\
& \left.+\beta_{u} U_{X_{u}}(s)\left\langle U_{Y_{u}}(s)+U_{Y_{e}}(s), f\right\rangle\right) \\
& \Psi_{Y_{e}}^{g}(U)(t)=\int_{0}^{t} d s\left(\alpha_{o n}\left\langle U_{Y_{u}}(s), g\right\rangle-\left\langle U_{Y_{e}}(s),\left(\alpha_{e n d}+\lambda_{e}\right) g\right\rangle\right. \\
& +U_{X_{e}}(s)\left\langle U_{Y_{u}}(s)+U_{Y_{e}}(s), \beta_{e} g\right\rangle \\
& \left.+\mu\left\langle U_{Y_{e}}(s), \int_{0}^{\infty} \rho(h) d h\left(\tau_{h} g-g\right)\right\rangle\right) \text {. }
\end{aligned}
$$

The map $\hat{\Psi}^{f, g}$ is defined from $D\left([0, T], E_{1}\right)$ into $D\left([0, T], \mathcal{S}_{5}\right)$, where $\mathcal{S}_{5}$ is the space of real $5 \times 5$ symmetric matrices, as follows. Let

$$
\hat{\psi}^{f, g}=\left(\begin{array}{ccccc}
\hat{\psi}_{X_{u}} & \hat{\psi}_{X_{u}, X_{r}} & \hat{\psi}_{X_{u}, X_{e}} & \hat{\psi}_{X_{u}, Y_{u}}^{f} & 0 \\
\hat{\psi}_{X_{u}, X_{r}} & \hat{\psi}_{X_{r}} & 0 & \hat{\psi}_{X_{r}, Y_{u}}^{f} & 0 \\
\hat{\psi}_{X_{u}, X_{e}} & 0 & \hat{\psi}_{X_{e}} & 0 & \hat{\psi}_{X_{e}, Y_{e}}^{g} \\
\hat{\psi}_{X_{u}, Y_{u}}^{f} & \hat{\psi}_{X_{r}, Y_{u}}^{f} & 0 & \hat{\psi}_{Y_{u}}^{f} & \hat{\psi}_{Y_{u}, g}^{f, Y_{e}} \\
0 & 0 & \hat{\psi}_{X_{e}, Y_{e}}^{g} & \hat{\psi}_{Y_{u}, Y_{e}}^{f, g} & \hat{\psi}_{Y_{e}}^{g}
\end{array}\right)
$$


with

$$
\begin{aligned}
& \left\{\begin{aligned}
& \begin{array}{rl}
\hat{\psi}_{X_{u}}(U(s))=\alpha_{o n} U_{X_{u}}(s)+ & \alpha_{r} U_{X_{r}}(s)+\alpha_{e n d} U_{X_{e}}(s) \\
& +\beta_{u} U_{X_{u}}(s)\left\langle U_{Y_{u}}(s)+U_{Y_{e}}(s), 1\right\rangle \\
\hat{\psi}_{X_{r}}(U(s))=\alpha_{r} U_{X_{r}}(s)+\lambda_{u}\left\langle U_{Y_{u}}(s), 1\right\rangle & \alpha_{\text {ond }} U_{X_{e}}(s)+\left\langle U_{Y_{e}}(s), \lambda_{e}\right\rangle \\
& +U_{X_{e}}(s)\left\langle U_{Y_{u}}(s)+U_{Y_{e}}(s), \beta_{e}\right\rangle
\end{array} \\
& \hat{\psi}_{Y_{u}}^{f}(U(s))=\alpha_{o n} U_{X_{u}}(s)+\left(\alpha_{o n}+\lambda_{u}\right)\left\langle U_{Y_{u}}(s), f^{2}\right\rangle+\alpha_{e n d}\left\langle U_{Y_{e}}(s), f^{2}\right\rangle \\
& \quad+\beta_{u} U_{X_{u}}(s)\left\langle U_{Y_{u}}(s)+U_{Y_{e}}(s), f^{2}\right\rangle \\
& \hat{\psi}_{Y_{e}}^{g}(U(s))=\alpha_{o n}\left\langle U_{Y_{u}}(s), g^{2}\right\rangle+\left\langle U_{Y_{e}}(s),\left(\alpha_{e n d}+\lambda_{e}\right) g^{2}\right\rangle \\
&+U_{X_{e}}(s)\left\langle U_{Y_{u}}(s)+U_{Y_{e}}(s), \beta_{e} g^{2}\right\rangle+\mu\left\langle U_{Y_{e}}(s), \int_{0}^{\infty} \rho(h) d h\left(\tau_{h} g-g\right)^{2}\right\rangle
\end{aligned}\right. \\
& \hat{\psi}_{X_{u}, X_{r}}(U(s))=-\alpha_{r} U_{X_{r}}(s) \\
& \hat{\psi}_{X_{u}, X_{e}}(U(s))=-\left(\alpha_{o n} U_{X_{u}}(s)+\alpha_{e n d} U_{X_{e}}(s)\right) \\
& \hat{\psi}_{X_{u}, Y_{u}}^{f}(U(s))=-\beta_{u} U_{X_{u}}(s)\left\langle U_{Y_{u}}(s)+U_{Y_{e}}(s), f\right\rangle \\
& \hat{\psi}_{X_{r}, Y_{u}}^{f}(U(s))=-\lambda_{u}\left\langle U_{Y_{u}}(s), f\right\rangle \\
& \hat{\psi}_{X_{e}, Y_{e}}^{g}(U(s))=-\left(\left\langle U_{Y_{e}}(s), \lambda_{e} g\right\rangle+U_{X_{e}}(s)\left\langle U_{Y_{u}}(s)+U_{Y_{e}}(s), \beta_{e} g\right\rangle\right) \\
& \hat{\psi}_{Y_{u}, Y_{e}}^{f, g}(U(s))=-\left(\alpha_{o n}\left\langle U_{Y_{u}}(s), f g\right\rangle+\alpha_{e n d}\left\langle U_{Y_{e}}(s), f g\right\rangle\right)
\end{aligned}
$$

Then $\hat{\Psi}(U)(t)=\int_{0}^{t} \hat{\psi}(U(s)) d s$, where integration is componentwise.

The following proposition states a semi-martingale decomposition which is at the heart of the proofs.

Proposition 3.1. For all measurable bounded $f, g: \mathbb{R}_{+} \rightarrow \mathbb{R}$, we may write

$$
Z^{n, f, g}=V^{n, f, g}+M^{n, f, g},
$$

where

$$
V^{n, f, g}(t)=Z^{n, f, g}(0)+\Psi^{f, g}\left(Z^{n}\right)(t)
$$

is a continuous finite variation process, and $M^{n, f, g}$ is a bounded càdlàg martingale, with predictable quadratic variation process

$$
\ll M^{n, f, g} \gg=\frac{1}{n} \hat{\Psi}^{f, g}\left(Z^{n}\right)
$$

Proof. Let $L^{n}$ denote the infinitesimal generator of the Markov process $Z^{n}$. By Ethier and Kurtz (2005, Proposition 4.1.7), for all measurable bounded function $\phi: E_{1} \rightarrow \mathbb{R}$, the process

$$
\phi\left(Z^{n}(t)\right)-\phi\left(Z^{n}(0)\right)-\int_{0}^{t} L^{n} \phi\left(Z^{n}(s)\right) d s
$$


is a bounded martingale. This implies in particular that $M^{n, f, g}$ is a martingale.

Taking $\phi\left(x_{1}, x_{2}, x_{3}, \nu_{1}, \nu_{2}\right)=x_{1}^{2}$, the process

$$
\begin{array}{rl}
U_{1}(t)=Z_{X_{u}}^{n}(t)^{2}- & Z_{X_{u}}^{n}(0)^{2} \\
-\int_{0}^{t} & d s\left(\alpha_{o n} Z_{X_{u}}^{n}(s)\left(\frac{1}{n}-2 Z_{X_{u}}^{n}(s)\right)+\alpha_{e n d} Z_{X_{e}}^{n}(s)\left(\frac{1}{n}+2 Z_{X_{u}}^{n}(s)\right)\right. \\
& +\alpha_{r} Z_{X_{r}}^{n}(s)\left(\frac{1}{n}+2 Z_{X_{u}}^{n}(s)\right) \\
& \left.+\beta_{u} Z_{X_{u}}^{n}(s)\left(\frac{1}{n}-2 Z_{X_{u}}^{n}(s)\right)\left\langle Z_{Y_{u}}^{n}(s)+Z_{Y_{e}}^{n}(s), 1\right\rangle\right)
\end{array}
$$

is thus a martingale.

Now, applying Itô's formula to the semi-martingale $Z_{X_{u}}^{n}$ and the function $x \mapsto x^{2}$, we find that the process

$$
\begin{array}{rl}
U_{2}(t)=Z_{X_{u}}^{n}(t)^{2}-Z_{X_{u}}^{n}(0)^{2} & \\
-2 \int_{0}^{t} & d s Z_{X_{u}}^{n}(s)\left(-\alpha_{o n} Z_{X_{u}}^{n}(s)+\alpha_{e n d} Z_{X_{e}}^{n}(s)+\alpha_{r} Z_{X_{r}}^{n}(s)\right. \\
& \left.\quad-\beta_{u} Z_{X_{u}}^{n}(s)\left\langle Z_{Y_{u}}^{n}(s)+Z_{Y_{e}}^{n}(s), 1\right\rangle\right)-<M_{X_{u}}^{n}>(t)
\end{array}
$$

is also a martingale. Since a finite variation predictable martingale is a.s. constant, we have $U_{2}-U_{1}=0$ a.s., hence the expression for $\left\langle M_{X_{u}}^{n}>\right.$.

The other components are found likewise, using appropriate functions.

\subsection{Convergence of the normalized process}

Since the predictable variation process $\left\langle M^{n, f, g} \gg\right.$ is a.s. $O(1 / n)$, it is reasonable to expect that the noise process $M^{n, f, g}$ will tend to 0 as $n \rightarrow \infty$, and that the normalized sequence will tend to a deterministic limit.

First we note that the initial condition vector, as defined in Section 2.1 . tends to a deterministic limit.

Lemma 3.2. As $n \rightarrow \infty, Z^{n}(0)$ tends to

$$
z(0)=\left(\theta_{X_{u}}, \theta_{X_{r}}, \theta_{X_{e}}, \theta_{Y_{u}} P_{u}, \theta_{Y_{e}} P_{e}\right)
$$

a.s. in $E_{1}$.

Theorem 3.3. For all $T>0$, the sequence $\left(Z^{n}\right)_{n \geqslant 1}$ converges in probability in $D\left([0, T], E_{1}\right)$ to the deterministic process $z=\left(x_{u}, x_{r}, x_{e}, \xi_{u}, \xi_{e}\right) \in C\left([0, T], E_{1}\right)$, which is the unique solution to the following equation

$$
z^{f, g}(t)=z^{f, g}(0)+\Psi^{f, g}(z)(t)
$$

for all measurable bounded functions $f, g: \mathbb{R}_{+} \rightarrow \mathbb{R}$. 
Proof. Since the limit is deterministic, it is enough to prove convergence in law.

The uniqueness of solutions, and the characterization of limit points of $\left(Z_{n}\right)_{n \geqslant 1}$ as solutions of (3.3p are established as in Fournier and Méléard (2004).

$C$-tightness (see Jacod and Shiryaev, 2003 , Definition VI.3.25) of the sequence $\left(Z^{n}\right)_{n \geqslant 1}$ in $D\left([0, T], E_{1}\right)$ is equivalent to $C$-tightness of each component (Jacod and Shiryaev, 2003, Corollary VI.3.33).

1. By Aldous' criterion Aldous, 1978), $\left(Z_{X_{u}}^{n}\right)_{n \geqslant 1},\left(Z_{X_{r}}^{n}\right)_{n \geqslant 1}$, and $\left(Z_{X_{e}}^{n}\right)_{n \geqslant 1}$ are tight in $D([0, T],[0,1])$. Since they have a.s. jumps of order $1 / n$ they are $C$-tight (Jacod and Shiryaev, 2003, Proposition VI.3.26).

2. Similarly, for all $f$ measurable and bounded, $\left(\left\langle Z_{Y_{u}}^{n}, f\right\rangle\right)_{n \geqslant 1}$ and $\left(\left\langle Z_{Y_{e}}^{n}, f\right\rangle\right)_{n \geqslant 1}$ are $C$-tight in $D([0, T], \mathbb{R})$. It follows that $\left(Z_{Y_{u}}^{n}\right)_{n \geqslant 1}$ and $\left(Z_{Y_{e}}^{n}\right)_{n \geqslant 1}$ are $C$-tight in $D\left([0, T], \mathcal{M}_{1}^{v}\right)$, where $\mathcal{M}_{1}^{v}$ denotes the space $\mathcal{M}_{1}$ endowed with the vague topology (Roelly-Coppoletta, 1986).

3. Thus $\left(Z^{n}\right)_{n \geqslant 1}$ is $C$-tight in $D\left([0, T], E_{1}^{v}\right)$, where $E_{1}^{v}=[0,1]^{3} \times\left(\mathcal{M}_{1}^{v}\right)^{2}$. If $\bar{Z}$ is a limit point in law of $\left(Z^{n}\right)_{n \geqslant 1}$ in $D\left([0, T], E_{1}^{v}\right)$, it can be shown that $\left\langle\bar{Z}_{Y_{u}}, 1\right\rangle$ and $\left\langle\bar{Z}_{Y_{e}}, 1\right\rangle$ are limit points in law in $D([0, T], \mathbb{R})$ of $\left(\left\langle Z_{Y_{u}}^{n}, 1\right\rangle\right)_{n \geqslant 1}$ and $\left(\left\langle Z_{Y_{u}}^{n}, 1\right\rangle\right)_{n \geqslant 1}$, respectively. The method of proof, similar to that in Méléard and Tran (2012), relies on approximating the functions $\mathbb{1}_{[k,+\infty)}$, $k \in \mathbb{N}$, by continuous functions such as

$$
\psi_{k}(m)= \begin{cases}0 & \text { if } m \leqslant k \\ m-k & \text { if } k<m \leqslant k+1 \\ 1 & \text { if } m>k+1\end{cases}
$$

noticing that the functions $1-\psi_{k}$ and $\int_{0}^{\infty} \rho(h) d h\left(\tau_{h} \psi_{k}-\psi_{k}\right)$ have compact support.

Theorem 3 in (Méléard and Roelly, 1993) then implies $C$-tightness of $\left(Z_{Y_{u}}^{n}\right)_{n \geqslant 1}$ and $\left(Z_{Y_{e}}^{n}\right)_{n \geqslant 1}$ in $D\left([0, T], \mathcal{M}_{1}\right)$.

\subsection{Absolute continuity and densities}

In this section, we assume that $P_{u}$ and $P_{e}$, hence $\xi_{u}(0)$ and $\xi_{e}(0)$, are absolutely continuous with respect to Lebesgue measure on $\mathbb{R}_{+}$.

Under these conditions, Gronwall's lemma implies that $\xi_{u}(t)$ and $\xi_{e}(t)$ are absolutely continuous for all $t$, and we let $\pi_{u}(t, m)$ and $\pi_{e}(t, m)$ denote their respective densities with respect to Lebesgue measure. The following corollary then follows from (3.3) and Fubini's theorem.

Theorem 3.4. Assume that $P_{u}$ and $P_{e}$ are absolutely continuous with respect to Lebesgue measure on $\mathbb{R}_{+}$, and that $\beta_{e}, \lambda_{e}$ and $\rho$ are bounded and continuous. 
Then $\left(x_{u}, x_{r}, y_{u}, \pi_{u}, \pi_{e}\right)$ is the solution to

$$
\left\{\begin{array}{c}
\frac{d x_{u}}{d t}=-\alpha_{o n} x_{u}+\alpha_{r} x_{r}+\alpha_{e n d} x_{e}-x_{u} \int_{0}^{\infty} \beta_{u}\left(\pi_{u}(t, m)+\pi_{e}(t, m)\right) d m \\
\frac{d x_{r}}{d t}=-\alpha_{r} x_{r}+\int_{0}^{\infty} \lambda_{u} \pi_{u}(t, m) d m \\
\frac{d x_{e}}{d t}=\alpha_{o n} x_{u}-\alpha_{e n d} x_{e}+\int_{0}^{\infty} \lambda_{e}(m) \pi_{e}(t, m) d m \\
\quad-x_{e} \int_{0}^{\infty} \beta_{e}(m)\left(\pi_{u}(t, m)+\pi_{e}(t, m)\right) d m \\
\begin{array}{r}
\frac{\partial}{\partial t} \pi_{u}(t, m)=-\left(\lambda_{u}+\alpha_{o n}\right) \pi_{u}(t, m)+x_{u} \beta_{u}\left(\pi_{u}(t, m)+\pi_{e}(t, m)\right) \\
\frac{\partial}{\partial t} \pi_{e}(t, m)=\alpha_{\text {on }} \pi_{u}(t, m)-\left(\alpha_{e n d}+\lambda_{e}(m)\right) \pi_{e}(t, m)
\end{array} \\
+x_{e} \beta_{e}(m)\left(\pi_{u}(t, m)+\pi_{e}(t, m)\right) \\
+\mu \int_{0}^{m} \pi_{e}(t, h) \rho(m-h) d h-\mu \pi_{e}(t, m)
\end{array}\right.
$$

with initial condition $\left(\theta_{X_{u}}, \theta_{X_{r}}, \theta_{X_{e}}, \theta_{Y_{u}} \frac{d P_{u}}{d m}, \theta_{Y_{e}} \frac{d P_{e}}{d m}\right)$.

This is the system of ordinary and partial integro-differential equations that was derived heuristically in Temime et al (2005). The main interest of the system of equations (3.4), in contrast to equation (3.3), is that it can be integrated numerically, and used to analyze the long term trends of antibiotic resistance. In this respect, Theorems 3.3 and 3.4 confer theoretical validity to the simulations presented in Temime et al (2005, section 5.3), which show that, in the long term, the respective numbers of individuals in the five compartments tend to a steady-state equilibrium, whereas the level of resistance in colonized individuals increases with time. Likewise, system (3.4) may be used to estimate the distribution of resistance levels in colonized individuals after a given period of time (Temime et al, 2005, section 6.2). The approximation of the stochastic model by its deterministic limit was observed to be acceptable for population sizes above 5000 .

\section{Central limit theorem}

In this section, we prove a central limit theorem for the sequence $\left(\eta^{n}\right)_{n \geqslant 1}=$ $\left(\sqrt{n}\left(Z^{n}-z\right)\right)_{n \geqslant 1}$ of fluctuation processes.

Writing as above $\eta^{n}=\left(\eta_{X_{u}}^{n}, \eta_{X_{r}}^{n}, \eta_{X_{e}}^{n}, \eta_{Y_{u}}^{n}, \eta_{Y_{e}}^{n}\right)$, we note that $\eta_{Y_{u}}^{n}(t)$ and $\eta_{Y_{e}}^{n}(t)$ are signed measures, whose limits, as $n \rightarrow \infty$, are not measure valued in general. Thus, following the approach of Métivier (1987) and Oelschläger $(1990)$, we are led to chose as state space for $\left(\eta^{n}(t)\right)_{t \geqslant 0}$ a space of distributions which is the dual of a weighted Sobolev space. 


\subsection{Notations, definitions, and preliminary results}

Although the level of resistance to antibiotics is a positive variable, it will be convenient to consider that the support of the measures $\eta_{Y_{u}}^{n}(t)$ and $\eta_{Y_{e}}^{n}(t)$ is the whole of $\mathbb{R}$. Likewise, we shall also consider that the support of the measures $P_{u}, P_{e}, Z_{Y_{u}}^{n}(t), Z_{Y_{e}}^{n}(t), \xi_{u}(t)$, and $\xi_{e}(t)$ is $\mathbb{R}$, and that the functions $\lambda_{e}$ and $\beta_{e}$ are defined on $\mathbb{R}$.

For $k \in \mathbb{N}$ and $\gamma \geqslant 0$, let $W^{k, \gamma}$ denote the space of functions $\phi: \mathbb{R} \rightarrow \mathbb{R}$, such that

$$
\|\phi\|_{W^{k, \gamma}}^{2}=\int_{\mathbb{R}} \sum_{j \leqslant k}\left|D^{j} \phi(m)\right|^{2} \frac{d m}{1+|m|^{2 \gamma}}<+\infty,
$$

where $D^{j} \phi$ is the weak $j$ th derivative of $\phi$. Equipped with the $\|\cdot\|_{W^{k, \gamma}}$ norm, $W^{k, \gamma}$ is a separable Hilbert space, whose dual is denoted by $W^{-k, \gamma}$, with dual norm $\|\cdot\|_{W^{-k, \gamma}}$. In the present context, the space of compactly supported smooth functions on $\mathbb{R}$ is dense in $W^{k, \gamma}$ (Kufner and Opic, 1984).

We also define the space $C^{k, \gamma}$ of $k$-times continuously differentiable functions on $\mathbb{R}$, such that

$$
\lim _{|m| \rightarrow \infty} \frac{\left|D^{j} f(m)\right|}{1+|m|^{\gamma}}=0 \quad \forall j \leqslant k,
$$

with norm

$$
\|f\|_{C^{k, \gamma}}=\sum_{j \leqslant k} \sup _{m \in \mathbb{R}} \frac{\left|D^{j} f(m)\right|}{1+|m|^{\gamma}} .
$$

Clearly, if $f \in C^{k, \gamma},|f(m)| \leqslant\|f\|_{C^{k, \gamma}}\left(1+|m|^{\gamma}\right)$. Moreover for $k \geqslant 0, l \geqslant 1$, $\gamma \geqslant 0$, and $\alpha>1 / 2$, the inclusions

$$
W^{k+l, \gamma} \hookrightarrow C^{k, \gamma} \hookrightarrow W^{k, \gamma+\alpha}
$$

are continuous (Métivier, 1987), i.e. there exist constants $K$ and $L$ such that $\|f\|_{W^{k, \gamma+\alpha}} \leqslant K\|f\|_{C^{k, \gamma}} \leqslant L\|f\|_{W^{k+l, \gamma}}$.

The following Proposition is proved as in Méléard (1998, Lemma A, section $3.2)$, using the continuity of the inclusion $W^{\overline{k, \gamma} \hookrightarrow} C^{0, \gamma}$ for $k \geqslant 1$.

Proposition 4.1. Let $k \in \mathbb{N}^{*}$ and $\gamma \geqslant 0$. For fixed $m \in \mathbb{R}$ and $h \geqslant 0$, the mappings

$$
\begin{aligned}
& D_{m}: \phi \in W^{k, \gamma} \mapsto \phi(m) \\
& D_{m h}: \phi \in W^{k, \gamma} \mapsto\left(\tau_{h} \phi-\phi\right)(m)
\end{aligned}
$$

are linear and continuous. Moreover

$$
\begin{aligned}
\left\|D_{m}\right\|_{W^{-k, \gamma}} & \leqslant K\left(1+|m|^{\gamma}\right) \\
\left\|D_{m h}\right\|_{W^{-k, \gamma}} & \leqslant K\left(1+|m|^{\gamma}+h^{\gamma}\right) .
\end{aligned}
$$

By Parseval's identity, we deduce 
Corollary 4.2. Let $\left(\phi_{j}\right)_{j \geqslant 1}$ be a Hilbert basis of $W^{k, \gamma}$. Then

$$
\begin{aligned}
& \sum_{j \geqslant 1} \phi_{j}(m)^{2} \leqslant K\left(1+|m|^{2 \gamma}\right) \\
& \sum_{j \geqslant 1}\left(\left(\tau_{h} \phi_{j}-\phi_{j}\right)(m)\right)^{2} \leqslant K\left(1+|m|^{2 \gamma}+h^{2 \gamma}\right) .
\end{aligned}
$$

For $k \in \mathbb{N}$ and $\gamma \geqslant 0$, let $\widetilde{E}^{-k, \gamma}=\mathbb{R}^{3} \times\left(W^{-k, \gamma}\right)^{2}$, equipped with the Hibertian product norm

$$
\|\zeta\|_{-k, \gamma}=\left(\left|\zeta_{X_{u}}\right|^{2}+\left|\zeta_{X_{r}}\right|^{2}+\left|\zeta_{X_{e}}\right|^{2}+\left\|\zeta_{Y_{u}}\right\|_{W^{-k, \gamma}}^{2}+\left\|\zeta_{Y_{e}}\right\|_{W^{-k, \gamma}}^{2}\right)^{1 / 2}
$$

The motivation for considering weighted Sobolev spaces is that we need constant functions to belong to the space of test functions. While any $\gamma>1 / 2$ would thus be adequate, we have chosen $\gamma=1$ for simplicity. The choice of $k$ was guided by two considerations: first, $k \geqslant 1$ is required for continuity of the map $m \mapsto \delta_{m}$; and second, tightness of $\left(\eta_{n}\right)_{n \geqslant 1}$ will be proved using the fact that the dual inclusion $W^{-k, \gamma+\alpha} \hookrightarrow W^{-(k+1), \gamma}$ is a Hilbert-Schmidt operator for $\alpha>1 / 2$. Overall this led to the choice of $W^{-2,1}$ as state space for $\eta_{Y_{u}}^{n}$ and $\eta_{Y_{e}}^{n}$, and of $W^{-1,1+\alpha}, \alpha>1 / 2$ as an auxiliary state space.

Now, the measures $Z_{Y_{u}}^{n}(t), Z_{Y_{e}}^{n}(t), \xi_{u}(t)$ and $\xi_{e}(t)$ belong to $W^{-1,1+\alpha}$ as soon as they have moments of order $q=2(1+\alpha)>3$. However, the existence of moments of order $q>5$ is needed in order to deal with the quadratic variation processes, whose expressions contain products of test functions.

For $p \geqslant 0$, let $\chi^{p}$ denote the function $m \mapsto|m|^{p}$, and let $\rho_{p}=\int_{0}^{\infty} h^{p} \rho(h) d h$.

Hypothesis 1. There exists $q>5$ such that

$$
\left\langle P_{u}, \chi^{q}\right\rangle<+\infty, \quad\left\langle P_{e}, \chi^{q}\right\rangle<+\infty, \quad \text { and } \quad \rho_{q}<+\infty .
$$

The proof of the following Lemma, which shows that the above moment hypothesis propagates in time, is analogous to that of Theorem 3.1 in Fournier and Méléard (2004).

Lemma 4.3. Under Hypothesis 1 .

$$
\begin{array}{r}
\sup _{0 \leqslant t \leqslant T}\left\langle\xi_{u}(t)+\xi_{e}(t), \chi^{q}\right\rangle<+\infty, \\
\sup _{n} \mathbb{E} \sup _{0 \leqslant t \leqslant T}\left\langle Z_{Y_{u}}^{n}(t)+Z_{Y_{e}}^{n}(t), \chi^{q}\right\rangle<+\infty .
\end{array}
$$

The above lemma shows in particular that the mappings $\Psi^{\phi, \psi}$ and $\hat{\Psi}^{\phi, \psi}$ are well defined for all $\phi, \psi \in W^{1, q}$.

We have the semimartingale decomposition

$$
\eta^{n, \phi, \psi}(t)=\widetilde{V}^{n, \phi, \psi}(t)+\widetilde{M}^{n, \phi, \psi}(t),
$$

where

$$
\begin{aligned}
\tilde{V}^{n, \phi, \psi}(t) & =\sqrt{n}\left(V^{n, \phi, \psi}(t)-z^{\phi, \psi}(t)\right) \\
& =\eta^{n, \phi, \psi}(0)+\sqrt{n}\left(\Psi^{\phi, \psi}\left(Z^{n}\right)(t)-\Psi^{\phi, \psi}(z)(t)\right)
\end{aligned}
$$


is a continuous finite variation process, and

$$
\widetilde{M}^{n, \phi, \psi}(t)=\sqrt{n} M^{n, \phi, \psi}(t)
$$

is a square integrable martingale with predictable quadratic variation process $\ll \widetilde{M}^{n, \phi, \psi} \gg=\hat{\Psi}^{\phi, \psi}\left(Z^{n}\right)$.

In obvious notations, we consider the processes $\widetilde{V}^{n}$ and $\widetilde{M}^{n}$, which, as shown below, may be considered as $\widetilde{E}^{-1, q / 2}$ valued processes.

\subsection{The martingale term}

Proposition 4.4. The process $\widetilde{M}^{n}=\left(\widetilde{M}_{X_{u}}^{n}, \widetilde{M}_{X_{r}}^{n}, \widetilde{M}_{X_{e}}^{n}, \widetilde{M}_{Y_{u}}^{n}, \widetilde{M}_{Y_{e}}^{n}\right)$ is a.s. a càdlàg square integrable martingale, taking its values in the Hilbert space $\widetilde{E}^{-1, q / 2}$. Moreover

$$
\sup _{n} \mathbb{E}\left(\sup _{0 \leqslant t \leqslant T}\left\|\widetilde{M}^{n}(t)\right\|_{-1, q / 2}^{2}\right)<+\infty .
$$

Proof. Let $\left(\phi_{j}\right)_{j \geqslant 1}$ be a Hilbert basis of $W^{1, q / 2}$. By Parseval's identity,

$$
\begin{aligned}
\left\|\widetilde{M}^{n}(t)\right\|_{-1, q / 2}^{2}=\left(\widetilde{M}_{X_{u}}^{n}(t)\right)^{2}+\left(\widetilde{M}_{X_{r}}^{n}(t)\right)^{2}+\left(\widetilde{M}_{X_{e}}^{n}(t)\right)^{2} & \\
& +\sum_{j \geqslant 1} \widetilde{M}_{Y_{u}}^{n, \phi_{j}}(t)^{2}+\sum_{j \geqslant 1} \widetilde{M}_{Y_{e}}^{n, \phi_{j}}(t)^{2} .
\end{aligned}
$$

By Doob's inequality, Proposition 3.1 and Corollary 4.2 , we have

$$
\begin{aligned}
\mathbb{E}\left(\sup _{0 \leqslant t \leqslant T} \widetilde{M}_{X_{u}}^{n}(t)^{2}+\sup _{0 \leqslant t \leqslant T} \widetilde{M}_{X_{r}}^{n}(t)^{2}+\sup _{0 \leqslant t \leqslant T} \widetilde{M}_{X_{e}}^{n}(t)^{2}\right. \\
\left.\quad+\sum_{j \geqslant 1} \sup _{0 \leqslant t \leqslant T} \widetilde{M}_{Y_{u}}^{n, \phi_{j}}(t)^{2}+\sum_{j \geqslant 1} \sup _{0 \leqslant t \leqslant T} \widetilde{M}_{Y_{e}}^{n, \phi_{j}}(t)^{2}\right) \\
<4 T\left(K_{1}+K_{2} \mathbb{E} \sup _{0 \leqslant t \leqslant T}\left\langle Z_{Y_{u}}^{n}+Z_{Y_{e}}^{n}, \chi^{q}\right\rangle\right)
\end{aligned}
$$

and the last line is bounded uniformly in $n$, by Hypothesis 1 and Lemma 4.3 .

Moreover $\widetilde{M}^{n}$ is a martingale in the separable Hilbert space $\widetilde{E}^{-1, q / 2}$, since $\widetilde{M}^{n, \phi_{j}, \phi_{k}}$ is a martingale for all $j$ and $k$, and $\mathbb{E}\left(\sup _{j, k}\left\|\widetilde{M}^{n, \phi_{j}, \phi_{k}}\right\|_{\mathbb{R}^{5}}\right)<\infty$ (see Badrikian, 1996)).

Finally, mimicking the proof of Ferland et al (1992, Proposition 3.5) shows that $\widetilde{M}^{n}$ has a.s. càdlàg paths.

The definitions of the scalar Doob-Meyer processes of a square integrable Hilbert-valued martingale may be found in (Badrikian, 1996) or (Métivier. 1982).

Proposition 4.5. The scalar Doob-Meyer process $\nless \widetilde{M}^{n} \ngtr$ of the square integrable martingale $\widetilde{M}^{n}$ is given by

$$
\nless \widetilde{M}^{n} \ngtr=<\widetilde{M}_{X_{u}}^{n}>+<\widetilde{M}_{X_{r}}^{n}>+<\widetilde{M}_{X_{e}}^{n}>+\nless \widetilde{M}_{Y_{u}}^{n} \ngtr+\nless \widetilde{M}_{Y_{e}}^{n} \ngtr
$$


where

$$
\begin{aligned}
\nless \widetilde{M}_{Y_{u}}^{n} \ngtr(t)=\int_{0}^{t} d s & \left(\left(\lambda_{u}+\alpha_{o n}\right)\left\langle Z_{Y_{u}}^{n}(s),\left\|D_{m}\right\|_{W^{-1, q / 2}}^{2}\right\rangle\right. \\
& +\beta_{u} Z_{X_{u}}^{n}(s)\left\langle Z_{Y_{u}}^{n}(s)+Z_{Y_{e}}^{n}(s),\left\|D_{m}\right\|_{W^{-1, q / 2}}^{2}\right\rangle \\
& \left.+\alpha_{e n d}\left\langle Z_{Y_{e}}^{n}(s),\left\|D_{m}\right\|_{W^{-1, q / 2}}^{2}\right\rangle\right)
\end{aligned}
$$

and

$$
\begin{aligned}
\nless \widetilde{M}_{Y_{e}}^{n} \ngtr(t)=\int_{0}^{t} d & \left(\alpha_{o n}\left\langle Z_{Y_{u}}^{n}(s),\left\|D_{m}\right\|_{W^{-1, q / 2}}^{2}\right\rangle\right. \\
& +\left\langle Z_{Y_{e}}^{n}(s),\left(\lambda_{e}+\alpha_{e n d}\right)\left\|D_{m}\right\|_{W^{-1, q / 2}}^{2}\right\rangle \\
& +Z_{X_{e}}^{n}(s)\left\langle Z_{Y_{u}}^{n}(s)+Z_{Y_{e}}^{n}(s), \beta_{e}\left\|D_{m}\right\|_{W^{-1, q / 2}}^{2}\right\rangle \\
& \left.+\left\langle Z_{Y_{e}}^{n}(s), \mu\left\|D_{m h}\right\|_{W^{-1, q / 2}}^{2}\right\rangle\right) .
\end{aligned}
$$

\subsection{The drift term}

We now consider the continuous finite variation process $\tilde{V}^{n}$.

The following assumption ensures that the mappings $\phi \mapsto \lambda_{e} \phi$ and $\phi \mapsto \beta_{e} \phi$ are bounded operators from $W^{1, q / 2}$ to $W^{1, q / 2}$, and from $W^{2,1}$ to $W^{2,1}$.

Hypothesis 2. The functions $\lambda_{e}$ and $\beta_{e}$ are twice differentiable, and their derivatives are bounded a.e.

Lemma 4.6. Let $T>0$. Under Hypotheses 1 and 2 ,

$$
\left\|\tilde{V}^{n}(t)\right\|_{-1, q / 2} \leqslant\left\|\eta^{n}(0)\right\|_{-1, q / 2}+\mathbf{J}_{T} \int_{0}^{t} d s\left\|\eta^{n}(s)\right\|_{-1, q / 2}
$$

where $\mathbf{J}_{T}$ is a deterministic constant that is independent of $n$ and $t$ for $t \leqslant T$.

Proof. Writing $v^{n}=\widetilde{V}^{n}-\eta^{n}(0)$, we have

$$
\begin{aligned}
\left\|\tilde{V}^{n}(t)\right\|_{-1, q / 2} \leqslant\left\|\eta^{n}(0)\right\|_{-1, q / 2} & +\left\|v^{n}(t)\right\|_{-1, q / 2} \\
\leqslant\left\|\eta^{n}(0)\right\|_{-1, q / 2}+ & \left|v_{X_{u}}^{n}(t)\right|+\left|v_{X_{r}}^{n}(t)\right|+\left|v_{X_{e}}^{n}(t)\right| \\
& +\left\|v_{Y_{u}}^{n}(t)\right\|_{W^{-1, q / 2}}+\left\|v_{Y_{e}}^{n}(t)\right\|_{W^{-1, q / 2}} .
\end{aligned}
$$


Let $\phi, \psi \in W^{1, q / 2}$. Since $v^{n, \phi, \psi}=\sqrt{n}\left(\Psi^{\phi, \psi}\left(Z^{n}\right)-\Psi^{\phi, \psi}(z)\right)$, we get

$$
\begin{aligned}
& \left|v_{X_{u}}^{n}(t)\right| \leqslant \int_{0}^{t} d s\left(\alpha_{o n}\left|\eta_{X_{u}}^{n}(s)\right|+\alpha_{r}\left|\eta_{X_{r}}^{n}(s)\right|+\alpha_{e n d}\left|\eta_{X_{e}}^{n}(s)\right|\right. \\
& \left.+\left(\left\|\eta_{Y_{u}}^{n}(s)\right\|_{W^{-1, q / 2}}+\left\|\eta_{Y_{e}}^{n}(s)\right\|_{W^{-1, q / 2}}\right)\left\|\beta_{u}\right\|_{W^{1, q / 2}}+\beta_{u}\left|\eta_{X_{u}}^{n}(s)\right|\right) \\
& \left|v_{X_{r}}^{n}(t)\right| \leqslant \int_{0}^{t} d s\left(\alpha_{r}\left|\eta_{X_{r}}^{n}(s)\right|+\left\|\eta_{Y_{u}}^{n}(s)\right\|_{W^{-1, q / 2}}\left\|\lambda_{u}\right\|_{W^{1, q / 2}}\right) \\
& \left|v_{X_{e}}^{n}(t)\right| \leqslant \int_{0}^{t} d s\left(\alpha_{o n}\left|\eta_{X_{u}}^{n}(s)\right|+\alpha_{e n d}\left|\eta_{X_{e}}^{n}(s)\right|+\left\|\eta_{Y_{e}}^{n}\right\|_{W^{-1, q / 2}}\left\|\lambda_{e}\right\|_{W^{1, q / 2}}\right. \\
& \left.+\left(\left\|\eta_{Y_{u}}^{n}(s)\right\|_{W^{-1, q / 2}}+\left\|\eta_{Y_{e}}^{n}(s)\right\|_{W^{-1, q / 2}}\right)\left\|\beta_{e}\right\|_{W^{1, q / 2}}+\bar{\beta}_{e}\left|\eta_{X_{e}}^{n}(s)\right|\right) \\
& \left|v_{Y_{u}}^{n, \phi}(t)\right| \leqslant \int_{0}^{t} d s\left(\left(\alpha_{o n}+\lambda_{u}\right)\left\|\eta_{Y_{u}}^{n}(s)\right\|_{W^{-1, q / 2}}\|\phi\|_{W^{1, q / 2}}\right. \\
& +\alpha_{e n d}\left\|\eta_{Y_{e}}^{n}(s)\right\|_{W^{-1, q / 2}}\|\phi\|_{W^{1, q / 2}} \\
& +\beta_{u}\left(\left\|\eta_{Y_{u}}^{n}(s)\right\|_{W^{-1, q / 2}}+\left\|\eta_{Y_{e}}^{n}(s)\right\|_{W^{-1, q / 2}}\right)\|\phi\|_{W^{1, q / 2}} \\
& \left.+\beta_{u}\left|\eta_{X_{u}}^{n}(s)\right|\left\|\xi_{u}(s)+\xi_{e}(s)\right\|_{W^{-1, q / 2}}\|\phi\|_{W^{1, q / 2}}\right) \\
& \left|v_{Y_{e}}^{n, \psi}(t)\right| \leqslant \int_{0}^{t} d s\left(\alpha_{o n}\left\|\eta_{Y_{u}}^{n}(s)\right\|_{W^{-1, q / 2}}\|\psi\|_{W^{1, q / 2}}\right. \\
& +\left\|\eta_{Y_{e}}^{n}(s)\right\|_{W^{-1, q / 2}}\left\|\left(\alpha_{e n d}+\lambda_{e}\right) \psi\right\|_{W^{1, q / 2}} \\
& +\left(\left\|\eta_{Y_{u}}^{n}(s)\right\|_{W^{-1, q / 2}}+\left\|\eta_{Y_{e}}^{n}(s)\right\|_{W^{-1, q / 2}}\right)\left\|\beta_{e} \psi\right\|_{W^{1, q / 2}} \\
& +\left|\eta_{X_{e}}^{n}(s)\right|\left\|\xi_{u}(s)+\xi_{e}(s)\right\|_{W^{-1, q / 2}}\left\|\beta_{e} \psi\right\|_{W^{1, q / 2}} \\
& \left.+\mu\left\|\eta_{Y_{e}}^{n}(s)\right\|_{W^{-1, q / 2}}\left\|\int_{-\infty}^{\infty} \rho(h) d h\left(\tau_{h} \psi-\psi\right)\right\|_{W^{1, q / 2}}\right) .
\end{aligned}
$$

Now observe that

- Constants $\beta_{u}$ and $\lambda_{u}$ belong to $W^{1, q / 2}$.

- By Hypothesis 2, functions $\beta_{e}$ and $\lambda_{e}$ belong to $W^{1, q / 2}$, and there exist constants $K_{\beta}$ and $K_{\lambda}$ such that $\left\|\beta_{e} \phi\right\|_{W^{1, q / 2}} \leqslant K_{\beta}\|\phi\|_{W^{1, q / 2}}$ and $\left\|\lambda_{e} \phi\right\|_{W^{1, q / 2}} \leqslant K_{\lambda}\|\phi\|_{W^{1, q / 2}}$ for all $\phi \in W^{1, q / 2}$.

- By Hypothesis 1, there exists a constant $K_{q / 2}$ such that $\left\|\int_{-\infty}^{\infty} \rho(h) d h \tau_{h} \psi\right\|_{W^{1, q / 2}} \leqslant K_{q / 2}\|\psi\|_{W^{1, q / 2}}$ for all $\psi \in W^{1, q / 2}$.

- By continuity of the inclusion $W^{1, q / 2} \hookrightarrow C^{0, q / 2}$ and Hypothesis 1 .

$$
\Xi_{q / 2}(T)=\sup _{0 \leqslant s \leqslant T}\left\|\xi_{u}(s)+\xi_{e}(s)\right\|_{W^{-1, q / 2}}<+\infty .
$$

This proves the Lemma with e.g.

$$
\begin{aligned}
\mathbf{J}_{T}=2\left(\alpha_{\text {on }}+\alpha_{r}+\alpha_{\text {end }}\right) & \\
& +\beta_{u}+\bar{\beta}_{e}+\left(\beta_{u}+K_{\beta}\right)\left(1+\Xi_{q / 2}(T)\right)+\left\|\beta_{u}\right\|_{W^{1, q / 2}}+\left\|\beta_{e}\right\|_{W^{1, q / 2}} \\
& +\lambda_{u}+K_{\lambda}+\left\|\lambda_{u}\right\|_{W^{1, q / 2}}+\left\|\lambda_{e}\right\|_{W^{1, q / 2}}+\mu\left(1+K_{q / 2}\right) .
\end{aligned}
$$


Lemma 4.7. Under Hypothesis 1 .

$$
\sup _{n} \mathbb{E}\left(\left\|\eta^{n}(0)\right\|_{-1, q / 2}^{2}\right)<+\infty
$$

Proof. Let $\left(\phi_{j}\right)_{j \geqslant 1}$ be a Hilbert basis in $W^{1, q / 2}$. By independence of the number $N_{Y_{u}}(0)$ of colonized unexposed individuals at time 0 , and their resistance levels $\left(a_{i}\right)_{i \geqslant 1}$, we have

$$
\begin{array}{r}
\mathbb{E}\left\|\eta_{Y_{u}}^{n}(0)\right\|_{W^{-1, q / 2}}^{2}=\frac{1}{n} \sum_{j \geqslant 1} \mathbb{E}\left\{\left(\sum_{i=1}^{N_{Y_{u}}(0)}\left(\phi_{j}\left(a_{i}\right)-\left\langle P_{u}, \phi_{j}\right\rangle\right)\right)^{2}\right\} \\
+n \mathbb{E}\left\{\left(\frac{N_{Y_{u}}(0)}{n}-\theta_{Y_{u}}\right)^{2}\right\} \sum_{j \geqslant 1}\left\langle P_{u}, \phi_{j}\right\rangle^{2}
\end{array}
$$

since the crossproducts are 0 . Indeed

$$
\begin{aligned}
\mathbb{E}[ & \left.\sum_{i=1}^{N_{Y_{u}}(0)}\left(\phi_{j}\left(a_{i}\right)-\left\langle P_{u}, \phi_{j}\right\rangle\right)\left(\frac{N_{Y_{u}}(0)}{n}-\theta_{Y_{u}}\right)\right] \\
& =\sum_{i=1}^{n} \mathbb{E}\left\{\mathbb{E}\left[\mathbb{1}_{\left\{i \leqslant N_{Y_{u}}(0)\right\}}\left(\phi_{j}\left(a_{i}\right)-\left\langle P_{u}, \phi_{j}\right\rangle\right)\left(\frac{N_{Y_{u}}(0)}{n}-\theta_{Y_{u}}\right)\right] \mid N_{Y_{u}}(0)\right\} \\
& \left.=\sum_{i=1}^{n} \mathbb{E}\left\{\left(\frac{N_{Y_{u}}(0)}{n}-\theta_{Y_{u}}\right) \mathbb{1}_{\left\{i \leqslant N_{Y_{u}}(0)\right\}} \mathbb{E}\left(\phi_{j}\left(a_{i}\right)-\left\langle P_{u}, \phi_{j}\right\rangle\right)\right]\right\} \\
& =0
\end{aligned}
$$

Now, by independence again,

$$
\begin{aligned}
\frac{1}{n} \sum_{j \geqslant 1} \mathbb{E}\left\{\left(\sum_{i=1}^{N_{Y_{u}}(0)}\left(\phi_{j}\left(a_{i}\right)-\left\langle P_{u}, \phi_{j}\right\rangle\right)\right)^{2}\right\} \\
=\frac{1}{n} \sum_{j \geqslant 1} \mathbb{E}\left(\sum_{i=1}^{N_{Y_{u}}(0)}\left(\phi_{j}\left(a_{i}\right)-\left\langle P_{u}, \phi_{j}\right\rangle\right)^{2}\right) \\
\leqslant \mathbb{E} \sum_{j \geqslant 1} \phi_{j}^{2}\left(a_{1}\right) \leqslant K \mathbb{E}\left(1+\left|a_{1}\right|^{q}\right),
\end{aligned}
$$

and

$$
\begin{aligned}
n \mathbb{E}\left\{\left(\frac{N_{Y_{u}}(0)}{n}-\theta_{Y_{u}}\right)^{2}\right\} \sum_{j \geqslant 1}\left\langle P_{u}, \phi_{j}\right\rangle^{2} & =\theta_{Y_{u}}\left(1-\theta_{Y_{u}}\right) \sum_{j \geqslant 1}\left\langle P_{u}, \phi_{j}\right\rangle^{2} \\
& \leqslant \theta_{Y_{u}}\left(1-\theta_{Y_{u}}\right) \mathbb{E}\left\|D_{a_{1}}\right\|_{W^{-1, q / 2}}^{2} .
\end{aligned}
$$

A similar bound holds for $\mathbb{E}\left\|\eta_{Y_{e}}^{n}(0)\right\|_{W^{-1, q / 2}}^{2}$. 


\subsection{Uniform estimate for the fluctuation process}

Proposition 4.8. Under Hypothesis 1.

$$
\begin{aligned}
& \sup _{n} \mathbb{E} \sup _{0 \leqslant t \leqslant T}\left\|\eta^{n}(t)\right\|_{-1, q / 2}^{2}<+\infty \\
& \sup _{n} \mathbb{E} \sup _{0 \leqslant t \leqslant T}\left\|\eta^{n}(t)\right\|_{-2,1}^{2}<+\infty
\end{aligned}
$$

Proof. By convexity, we have from 4.4

$$
\begin{aligned}
\left\|\eta^{n}(t)\right\|_{-1, q / 2}^{2} & \leqslant 2\left\|\tilde{V}^{n}(t)\right\|_{-1, q / 2}^{2}+2\left\|\widetilde{M}^{n}(t)\right\|_{-1, q / 2}^{2} \\
& \leqslant 4\left\|\eta^{n}(0)\right\|_{-1, q / 2}^{2}+4 \mathbf{J}_{T}^{2} t \int_{0}^{t} d s\left\|\eta^{n}(s)\right\|_{-1, q / 2}^{2}+2\left\|\widetilde{M}^{n}(t)\right\|_{-1, q / 2}^{2} .
\end{aligned}
$$

Thus, we get by Gronwall's lemma

$$
\begin{aligned}
\sup _{n} \mathbb{E} \sup _{0 \leqslant t \leqslant T} & \left\|\eta^{n}(t)\right\|_{-1, q / 2}^{2} \\
& \leqslant\left(4 \sup _{n} \mathbb{E}\left\|\eta^{n}(0)\right\|_{-1, q / 2}^{2}+2 \sup _{n} \mathbb{E} \sup _{0 \leqslant t \leqslant T}\left\|\widetilde{M}^{n}(t)\right\|_{-1, q / 2}^{2}\right) e^{4 \mathbf{J}_{T}^{2} T^{2}}
\end{aligned}
$$

which is finite by 4.2 and 4.5 . Inequality 4.7 follows by continuity of the inclusion $W^{-1, q / 2} \hookrightarrow W^{-2,1}$.

\subsection{Tightness in $D\left([0, T], \widetilde{E}^{-2,1}\right)$}

Proposition 4.9. The sequences $\left(\eta^{n}\right)_{n \geqslant 1}$ and $\left(\widetilde{M}^{n}\right)_{n \geqslant 1}$ are tight in $D\left([0, T], \widetilde{E}^{-2,1}\right)$.

Proof. To show that the sequences $\left(\widetilde{M}^{n}\right)_{n \geqslant 1}$ and $\left(\eta^{n}\right)_{n \geqslant 1}$ are tight in $D\left([0, T], \widetilde{E}^{-2,1}\right)$, we use the Aldous-Rebolledo-Joffe-Métivier criterion (Joffe and Métivier, 1986; Rebolledo, 1980) for Hilbert valued càdlàg semimartingales. First (4.2) and (4.6), together with the fact that the inclusion $\widetilde{E}^{-1, q / 2} \hookrightarrow \widetilde{E}^{-2,1}$ is a Hilbert-Schmidt operator, imply that, for fixed $t \in[0, T],\left(\widetilde{M}^{n}(t)\right)_{n \geqslant 1}$ and $\left(\eta^{n}(t)\right)_{n \geqslant 1}$ are tight in $\widetilde{E}^{-2,1}$. It remains to prove the Aldous conditions for $\nless \widetilde{M}^{n} \ngtr$ and for $\widetilde{V}^{n}$, which are easily established using Lemma 4.3

\subsection{Limit points}

Lemma 4.10. All limit points of $\left(\eta^{n}\right)_{n \geqslant 1}$ and $\left(\widetilde{M}^{n}\right)_{n \geqslant 1}$ are a.s. continuous.

Proof. Let $\Delta \eta^{n}(s)$ (respectively $\Delta \widetilde{M}^{n, \phi, \psi}(s)$ ) denote the jump at $s$ of $\eta^{n, \phi, \psi}$ (respectively $\left.\widetilde{M}^{n, \phi, \psi}\right)$. Since $\widetilde{M}^{n}$ and $\eta^{n}$ have the same jumps, it is enough to show that $\sup _{0 \leqslant t \leqslant T}\left\|\eta^{n}(t)-\eta^{n}(t-)\right\|_{-2,1}$ tends to 0 in probability (Jacod and Shiryaev, 2003, Proposition VI.3.26).

The jumps of $\eta_{X_{u}}^{n}, \eta_{X_{r}}^{n}$, and $\eta_{X_{e}}^{n}$, are a.s. of size $\frac{1}{\sqrt{n}}$. Let now $\phi \in W^{2,1}$, and consider the jumps of $\eta_{Y_{u}}^{n, \phi}$ and $\eta_{Y_{e}}^{n, \phi}$. Given an arbitrary, but fixed, enumeration 
$1, \cdots, n$ of the individuals in the population, we denote by $m_{i, n}(t)$ the resistance level of individual $i$ at time $t$, setting $m_{i, n}(t)=0$ if $i$ is uncolonized at $t$. Then, noting that a.s. two or more individuals cannot jump at the same time, the sizes of the possible jumps at time $t$ of $\eta_{Y_{u}}^{n, \phi}$ and $\eta_{Y_{e}}^{n, \phi}$ are

- $\left|\phi\left(m_{i, n}\left(t_{-}\right)\right)\right| / \sqrt{n}$ if: individual $i$ is colonized at $t_{-}$, and either undergoes decolonization, or a change in exposure status, or contaminates an uncolonized individual,

- $\left|\phi\left(m_{i, n}\left(t_{-}\right)+h\right)-\phi\left(m_{i, n}\left(t_{-}\right)\right)\right| / \sqrt{n}$ : if individual $i$ is exposed and colonized at $t_{-}$, and undergoes a mutation of amplitude $h$.

Thus in all instances the size of the jump is less than $\frac{K}{\sqrt{n}}\left(1+\left|m_{i, n}(t)\right|+h\right)$.

To obtain an uniform estimate for $\left|m_{i, n}(t)\right|$, let $\mathbb{1}_{\{j \in C\}}(t)=1$ if individual $j$ is in compartment $C$ at time $t$, and 0 otherwise, and let $k \in\{1, \ldots, n\}$ be an individual of the population. Taking in account only those transitions that increase the resistance level of individual $k$, we have

$$
\begin{aligned}
m_{k, n}^{2}(t) \leqslant & m_{k, n}^{2}(0) \\
+ & \int_{0}^{t} \int_{\mathbb{N}^{*}} \int_{\mathbb{N}^{*}} \mathbb{1}_{\left\{k \in X_{u}\right\}}\left(s_{-}\right) \mathbb{1}_{\{i=k\}} \mathbb{1}_{\left\{j \in Y_{u} \cup Y_{e}\right\}}\left(s_{-}\right) \\
& \times\left(m_{j, n}^{2}\left(s_{-}\right)-m_{k, n}^{2}\left(s_{-}\right)\right) Q_{X_{u}}^{c o l}(d s, d i, d j) \\
+ & \int_{0}^{t} \int_{\mathbb{N}^{*}} \int_{\mathbb{N}^{*}} \int_{0}^{1} \mathbb{1}_{\left\{k \in X_{e}\right\}}\left(s_{-}\right) \mathbb{1}_{\{i=k\}} \mathbb{1}_{\left\{j \in Y_{u} \cup Y_{e}\right\}}\left(s_{-}\right) \mathbb{1}_{\left\{v \leqslant \beta_{e}\left(m_{j, n}\left(s_{-}\right) / \bar{\beta}_{e}\right\}\right.} \\
+ & \int_{0}^{t} \int_{\mathbb{N}^{*}} \int_{0}^{\infty} \mathbb{1}_{\left\{k \in Y_{e}\right\}}\left(s_{-, n}^{2}\left(s_{-}\right)-m_{\{i=k\}}^{2}\left(s_{-}\right)\right) Q_{X_{e}}^{c o l}(d s, d i, d j, d v) \\
\leqslant & m_{k, n}^{2}(0)+\int_{0}^{t} \int_{\mathbb{N}^{*}} \int_{\mathbb{N}^{*}} \mathbb{1}_{\{i=k\}} \mathbb{1}_{\left\{j \in Y_{u} \cup m_{j, n}\left(s_{-}\right) / \bar{\mu}\right\}}\left(s_{-}\right) m_{j, n}^{2}\left(s_{-}\right) Q_{X_{u}}^{c o l}(d s, d i, d j) \\
& +\int_{0}^{t} \int_{\mathbb{N}^{*}} \int_{\mathbb{N}^{*}} \int_{0}^{1} \mathbb{1}_{\{i=k\}} \mathbb{1}_{\left\{j \in Y_{u} \cup Y_{e}\right\}}\left(s_{-}\right) m_{j, n}^{2}\left(s_{-}\right) Q_{X_{e}}^{c o l}(d s, d i, d j, d v) \\
& +K \int_{0}^{t} \int_{\mathbb{N}^{*}}^{\infty} \int_{0}^{\infty} \mathbb{1}_{\{i=k\}}\left(m_{k, n}^{2}\left(s_{-}\right)+h^{2}\right) Q^{m u t}(d s, d i, d h),
\end{aligned}
$$

whence

$$
\begin{aligned}
\mathbb{E}\left(\sup _{0 \leqslant t \leqslant T} m_{k, n}^{2}(t)\right) \leqslant\left\langle\theta_{Y_{u}} P_{u}+\theta_{Y_{e}} P_{e}, \chi^{2}\right\rangle & \\
& +\left(\beta_{u}+\bar{\beta}_{e}\right) \int_{0}^{T} d s \mathbb{E}\left(\sup _{0 \leqslant u \leqslant s}\left\langle Z_{Y_{u}}^{n}(u)+Z_{Y_{e}}^{n}(u), \chi^{2}\right\rangle\right) \\
& +K \mu \int_{0}^{T} d s \mathbb{E}\left(\sup _{0 \leqslant u \leqslant s} m_{k, n}^{2}(u)\right)+K \mu T \rho_{2} .
\end{aligned}
$$


Thus, by Lemma 4.3 and Gronwall's lemma,

$$
\sup _{n} \max _{1 \leqslant k \leqslant n} \mathbb{E}\left(\sup _{0 \leqslant t \leqslant T} m_{k, n}^{2}(t)\right)<+\infty,
$$

yielding

$$
\mathbb{E} \sup _{0 \leqslant t \leqslant T}\left\|\eta^{n}(t)-\eta^{n}(t-)\right\|_{-2,1}^{2} \leqslant \frac{K}{n},
$$

where $K$ is independent of $n$ and $i$. The Markov inequality concludes the proof.

Proposition 4.11. For all $T>0$, the sequence $\left(\widetilde{M}^{n}\right)_{n \geqslant 1}$ tends in law in $D\left([0, T], \widetilde{E}^{-2,1}\right)$ to the process $B=\left(B_{X_{u}}, B_{X_{r}}, B_{X_{e}}, B_{Y_{u}}, B_{Y_{e}}\right)$, which is a continuous centered square integrable Gaussian martingale, such that, for all $\phi, \psi \in W^{2,1}$, the predictable quadratic variation process of $B^{\phi, \psi}$ is

$$
\ll B^{\phi, \psi} \gg=\hat{\Psi}^{\phi, \psi}(z) .
$$

Proof. Let $B$ be the limit in law in $D\left([0, T], \tilde{E}^{-2,1}\right)$ of a subsequence $\left(\widetilde{M}^{n}\right)_{n \geqslant 1}$. For $\phi, \psi \in W^{2,1}$, the subsequence $\left(\widetilde{M}^{n, \phi, \psi}\right)_{n \geqslant 1}$ tends in law to $B^{\phi, \psi}$ (by the continuous mapping theorem). Since the jumps of $\widetilde{M}^{n, \phi, \psi}$ are those of $\eta^{n, \phi, \psi}$, we have

$$
\mathbb{E} \sup _{s \leqslant t}\left\|\Delta \widetilde{M}^{n, \phi, \psi}(s)\right\|_{\mathbb{R}^{5}}^{2} \leqslant\left(3+\|\phi\|_{W^{2,1}}^{2}+\|\psi\|_{W^{2,1}}^{2}\right) \mathbb{E} \sup _{s \leqslant t}\left\|\Delta \eta^{n}(s)\right\|_{-2,1}^{2}
$$

so that $\sup _{s \leqslant t}\left\|\Delta \widetilde{M}^{n, \phi, \psi}(s)\right\|_{\mathbb{R}^{5}}^{2} \rightarrow 0$ in probability for all $t$ by 4.8 .

We deduce from (4.1) that $\phi, \psi \in W^{2,1} \Rightarrow \phi \psi \in W^{1, q / 2}$ for $q>5$. It then follows by 4.6 and Hypothesis 1 that $\ll \widetilde{M}^{n, \phi, \psi} \gg_{t}$ tends in probability to $\hat{\Psi}^{\phi, \psi}(z)(t)$ for all fixed $t$. Now, the process $\hat{\Psi}^{\phi, \psi}(z)$ is deterministic and 0 at time 0 . Moreover the matrix $\hat{\Psi}^{\phi, \psi}(z)(t)-\hat{\Psi}^{\phi, \psi}(z)(s)$ is symmetric and nonnegative for all $s<t$.

Therefore, using Pollard (1984, Theorem VIII.13), we conclude that $\left(\widetilde{M}^{n, \phi, \psi}\right)_{n \geqslant 1}$ tends in law to the continuous Gaussian centered martingale $B^{\phi, \psi}$ with predictable quadratic variation $\ll B^{\phi, \psi} \gg=\hat{\Psi}^{\phi, \psi}(z)$.

Next, the family $\left\{\widetilde{M}^{n}(t), t \in[0, T], n \geqslant 1\right\}$ of $\widetilde{E}^{-2,1}$-valued random variables is uniformly integrable by $(4.2)$. Therefore $B$ is a martingale with respect to the filtration it generates (Jacod and Shiryaev, 2003, Propositions IX.1.1 and IX.1.12). Moreover, by Doob's inequality, $\mathbb{E} \sup _{0 \leqslant t \leqslant T}\|B(t)\|_{-2,1}^{2}<+\infty$.

Theorem 4.12. For all $T>0$, the sequence of processes $\left(\eta^{n}\right)_{n \geqslant 1}$ tends in law in $D\left([0, T], \widetilde{E}^{-2,1}\right)$ to the process $\eta=\left(\eta_{X_{u}}, \eta_{X_{r}}, \eta_{X_{e}}, \eta_{Y_{u}}, \eta_{Y_{e}}\right) \in C\left([0, T], \widetilde{E}^{-2,1}\right)$, 
which is the unique solution of the system

$$
\left\{\begin{array}{l}
\eta_{X_{u}}(t)=\eta_{X_{u}}(0)+\int_{0}^{t} d s\left(-\alpha_{o n} \eta_{X_{u}}(s)+\alpha_{r} \eta_{X_{r}}(s)+\alpha_{e n d} \eta_{X_{e}}(s)\right. \\
\left.-x_{u}(s)\left\langle\eta_{Y_{u}}(s)+\eta_{Y_{e}}(s), \beta_{u}\right\rangle-\eta_{X_{u}}(s)\left\langle\xi_{u}(s)+\xi_{e}(s), \beta_{u}\right\rangle\right)+B_{X_{u}}(t) \\
\eta_{X_{r}}(t)=\eta_{X_{r}}(0)+\int_{0}^{t} d s\left(-\alpha_{r} \eta_{X_{r}}(s)+\left\langle\eta_{Y_{u}}(s), \lambda_{u}\right\rangle\right)+B_{X_{r}}(t) \\
\eta_{X_{e}}(t)=\eta_{X_{e}}(0)+\int_{0}^{t} d s\left(\alpha_{o n} \eta_{X_{u}}(s)-\alpha_{e n d} \eta_{X_{e}}(s)+\left\langle\eta_{Y_{e}}(s), \lambda_{e}\right\rangle\right. \\
\left.\quad-x_{e}(s)\left\langle\eta_{Y_{u}}(s)+\eta_{Y_{e}}(s), \beta_{e}\right\rangle-\eta_{X_{e}}(s)\left\langle\xi_{u}(s)+\xi_{e}(s), \beta_{e}\right\rangle\right)+B_{X_{e}}(t) \\
\eta_{Y_{u}}^{\phi}(t)=\eta_{Y_{u}}^{\phi}(0)+\int_{0}^{t} d s\left(-\left\langle\eta_{Y_{u}}(s),\left(\alpha_{o n}+\lambda_{u}\right) \phi\right\rangle+\left\langle\eta_{Y_{e}}(s), \alpha_{e n d} \phi\right\rangle\right. \\
\left.+x_{u}(s)\left\langle\eta_{Y_{u}}(s)+\eta_{Y_{e}}(s), \beta_{u} \phi\right\rangle+\eta_{X_{u}}(s)\left\langle\xi_{u}(s)+\xi_{e}(s), \beta_{u} \phi\right\rangle\right)+B_{Y_{u}}^{\phi}(t) \\
\eta_{Y_{e}}^{\psi}(t)=\eta_{Y_{e}}^{\psi}(0)+\int_{0}^{t} d s\left(\left\langle\eta_{Y_{u}}(s), \alpha_{o n} \psi\right\rangle-\left\langle\eta_{Y_{e}}(s),\left(\alpha_{e n d}+\lambda_{e}\right) \psi\right\rangle\right. \\
+x_{e}(s)\left\langle\eta_{Y_{u}}(s)+\eta_{Y_{e}}^{n}(s), \beta_{e} \psi\right\rangle+\eta_{X_{e}}(s)\left\langle\xi_{u}(s)+\xi_{e}(s), \beta_{e} \psi\right\rangle \\
\left.+\left\langle\eta_{Y_{e}}(s), \mu \int_{0}^{\infty} \rho(h) d h\left(\tau_{h} \psi-\psi\right)\right\rangle\right)+B_{Y_{e}}^{\psi}(t),
\end{array}\right.
$$

for all $\phi, \psi \in W^{2,1}$, where $B^{\phi, \psi}$ is the Gaussian martingale defined in Proposition 4.11 .

The proof rests on the three following lemmas.

Lemma 4.13. All limit points in law of $\left(\eta_{n}\right)_{n \geqslant 1}$ are weak solutions of the stochastic differential system (4.9).

Proof. Let $\eta$ be the limit in law of a subsequence $\left(\eta_{n}\right)_{n \geqslant 1}$. For $\phi, \psi \in W^{2,1}$, write 4.9 in the form

$$
\eta^{\phi, \psi}(t)=\eta^{\phi, \psi}(0)+\int_{0}^{t} d s L^{\phi, \psi}(s) \eta(s)+B^{\phi, \psi}(t),
$$

and let the mapping $\widetilde{\Psi}^{\phi, \psi}: D\left([0, T], \widetilde{E}^{-2,1}\right) \rightarrow D\left([0, T], \mathbb{R}^{5}\right)$ be defined by

$$
\tilde{\Psi}^{\phi, \psi}(\zeta)(t)=\zeta^{\phi, \psi}(0)+\int_{0}^{t} d s L^{\phi, \psi}(s) \zeta(s) .
$$

We have

$$
\begin{aligned}
\widetilde{M}^{n, \phi, \psi} & =\eta^{n, \phi, \psi}-\widetilde{V}^{n, \phi, \psi} \\
& =\left(\eta^{n, \phi, \psi}-\widetilde{\Psi}^{\phi, \psi}\left(\eta^{n}\right)\right)+\left(\widetilde{\Psi}^{\phi, \psi}\left(\eta^{n}\right)-\widetilde{V}^{n, \phi, \psi}\right) .
\end{aligned}
$$


On the one hand, $\eta^{n, \phi, \psi}-\widetilde{V}^{n, \phi, \psi}$ tends in law to $B^{\phi, \psi}$, by Proposition 4.11 On the other hand, since $\eta$ is continuous (Lemma 4.10, $\eta^{n, \phi, \psi}-\widetilde{\Psi}^{\phi, \psi}\left(\eta^{n}\right)$ tends in law to $\eta^{\phi, \psi}-\widetilde{\Psi}^{\phi, \psi}(\eta)$ by the continuous mapping theorem. We now show that $\widetilde{\Psi}^{\phi, \psi}\left(\eta^{n}\right)-\widetilde{V}^{n, \phi, \psi}$ tends to zero in probability. Since

$$
\begin{gathered}
\left\|\widetilde{\Psi}^{\phi, \psi}\left(\eta^{n}\right)(t)-\tilde{V}^{n, \phi, \psi}(t)\right\|_{\mathbb{R}^{5}} \leqslant \frac{1}{\sqrt{n}} \int_{0}^{t} d s\left(\left|\eta_{X_{u}}^{n}(s)\right|\left|\left\langle\eta_{Y_{u}}^{n}(s)+\eta_{Y_{e}}^{n}(s), \beta_{u}(1+\phi)\right\rangle\right|\right. \\
\left.+\left|\eta_{X_{e}}^{n}(s) \|\left\langle\eta_{Y_{u}}^{n}(s)+\eta_{Y_{e}}^{n}(s), \beta_{e}(1+\psi)\right\rangle\right|\right) \\
\leqslant \frac{2}{\sqrt{n}} \int_{0}^{t} d s\left\|\eta^{n}(s)\right\|_{-2,1}^{2}\left(\left\|\beta_{u}(1+\phi)\right\|_{W^{2,1}}+\left\|\beta_{e}(1+\psi)\right\|_{W^{2,1}}\right)
\end{gathered}
$$

we have, for all $\epsilon>0$,

$$
\begin{aligned}
\mathbb{P}\left(\sup _{0 \leqslant t \leqslant T}\left|\widetilde{\Psi}^{\phi, \psi}\left(\eta^{n}\right)(t)-\widetilde{V}^{n, \phi, \psi}(t)\right|>\epsilon\right) & \leqslant \frac{K T}{\epsilon \sqrt{n}} \sup _{n} \mathbb{E}\left(\sup _{0 \leqslant s \leqslant T}\left\|\eta^{n}(s)\right\|_{-2,1}^{2}\right) \\
& \rightarrow 0 \text { as } n \rightarrow \infty \text { by } 4.7 .
\end{aligned}
$$

Hence, $\eta^{n, \phi, \psi}-\widetilde{V}^{n, \phi, \psi}$ tends in law to $\eta^{\phi, \psi}-\widetilde{\Psi}^{\phi, \psi}(\eta)$ Jacod and Shiryaev, 2003 Lemma VI.3.31). The lemma follows by uniqueness of the limit in law.

Lemma 4.14. $\eta(0)$ is defined uniquely as the limit in law in $\widetilde{E}^{-2,1}$ of $\left(\eta^{n}(0)\right)_{n \geqslant 1}$.

Proof. Since $\eta$ is continuous, $\left(\eta^{n}(0)\right)_{n \geqslant 1}$ is tight by projection at time 0 . Now, for all $\phi, \psi \in W^{2,1}$, straightforward calculations using characteristic functions show that any convergent subsequence $\left(\eta^{n, \phi, \psi}(0)\right)_{n \geqslant 1}$ tends in law to a Gaussian random variable with zero expectation and variance matrix

$\Omega^{\phi, \psi}=$

$$
\left(\begin{array}{ccccc}
\theta_{X_{u}}\left(1-\theta_{X_{u}}\right) & -\theta_{X_{u}} \theta_{X_{r}} & -\theta_{X_{u}} \theta_{X_{e}} & -\theta_{X_{u}} \theta_{Y_{u}} \bar{\phi} & -\theta_{X_{u}} \theta_{Y_{e}} \bar{\psi} \\
& \theta_{X_{r}}\left(1-\theta_{X_{r}}\right) & -\theta_{X_{r}} \theta_{X_{e}} & -\theta_{X_{r}} \theta_{Y_{u}} \bar{\phi} & -\theta_{X_{r}} \theta_{Y_{e}} \bar{\psi} \\
& & \theta_{X_{e}}\left(1-\theta_{X_{e}}\right) & -\theta_{X_{e}} \theta_{Y_{u}} \bar{\phi} & -\theta_{X_{e}} \theta_{Y_{e}} \bar{\psi} \\
& & & \theta_{Y_{u}}\left(\bar{\phi}_{2}-\theta_{Y_{u}} \bar{\phi}^{2}\right) & -\theta_{X_{u}} \theta_{X_{e}} \bar{\psi} \bar{\psi} \\
& & & \theta_{Y_{e}}\left(\bar{\psi}_{2}-\theta_{Y_{e}} \bar{\psi}^{2}\right)
\end{array}\right)
$$

where $\bar{\phi}=\left\langle P_{u}, \phi\right\rangle, \bar{\phi}_{2}=\left\langle P_{u}, \phi^{2}\right\rangle, \bar{\psi}=\left\langle P_{e}, \psi\right\rangle$, and $\bar{\psi}_{2}=\left\langle P_{e}, \psi^{2}\right\rangle$.

Lemma 4.15. Equation (4.9) has a unique solution in $D\left([0, T], \widetilde{E}^{-2,1}\right)$.

Proof. By Gronwall's lemma, the solution is pathwise unique. Next, by Jacod and Shiryaev (2003, Proposition IX.1.12), $B$ is a martingale with respect to the filtration generated by $(\eta, B)$, so that pathwise uniqueness implies uniqueness in law by the Yamada-Watanabe theorem (Revuz and Yor, 1991, Theorem IX.1.7). 


\section{$5 \quad$ Statistical applications}

The application of Theorem 4.12 to practical statistical issues is far from straightforward, due to the fact that the limit processes $z(t)$ and $\eta(t)$ have measure and distribution valued components, respectively. Moreover, although these processes may be converted to processes with real valued components by considering $z^{\phi, \psi}$ and $\eta^{\phi, \psi}$ for given $\phi, \psi \in W^{2,1}$, the latter processes cannot be computed nor simulated from (3.3) and (4.9) in general. In this sense, theorems 3.3 and 4.12 are essentially existence theorems.

However some results can be derived under more specific assumptions.

Proposition 5.1. Assume that the functions $\lambda_{e}$ and $\beta_{e}$ are constant. Then we have

$$
\eta^{1,1}(t)=E(t)\left(\eta^{1,1}(0)+\int_{0}^{t} E(s)^{-1} d B^{1,1}(s)\right)
$$

where

$$
a(t)=\left(\begin{array}{ccccc}
-\alpha_{o n} & \alpha_{r} & \alpha_{\text {end }} & -\beta_{u} x_{u}(t) & -\beta_{u} x_{u}(t) \\
-\beta_{u} \nu(t) & & & & \\
0 & -\alpha_{r} & 0 & \lambda_{u} & 0 \\
\alpha_{o n} & 0 & -\alpha_{\text {end }} & -\beta_{e} x_{e}(t) & -\beta_{e} x_{e}(t)+\lambda_{e} \\
& & -\beta_{e} \nu(t) & & \\
\beta_{u} \nu(t) & 0 & 0 & -\left(\alpha_{\text {on }}+\lambda_{u}\right) & \alpha_{\text {end }} \\
& & & +\beta_{u} x_{u}(t) & +\beta_{u} x_{u}(t) \\
0 & 0 & \beta_{e} \nu(t) & \alpha_{o n}+\beta_{e} x_{e}(t) & -\left(\alpha_{\text {end }}+\lambda_{e}\right) \\
& & & & +\beta_{e} x_{e}(t)
\end{array}\right)
$$

with $\nu(t)=\left\langle\xi_{u}(t)+\xi_{e}(t), 1\right\rangle$, and $E(t)=\exp \left(\int_{0}^{t} a(s) d s\right)$.

Proof. It readily follows from the assumption and 4.9 that $\eta^{1,1}(t)$ is solution to the Langevin equation

$$
\eta^{1,1}(t)=\eta^{1,1}(0)+\int_{0}^{t} a(s) \eta^{1,1}(s) d s+B^{1,1}(t)
$$

whose solution is (5.1).

Recall the definition of $\hat{\psi}^{f, g}(U(s))$ given by equations (3.1), and let $A^{*}$ denote the transpose of a square matrix $A$.

Corollary 5.2. If the functions $\lambda_{e}$ and $\beta_{e}$ are constant, $\sqrt{n}\left(Z^{n, 1,1}(t)-z^{1,1}(t)\right)$ tends in law to the Normal distribution on $\mathbb{R}^{5}$ with zero expectation and variance matrix

$$
\Sigma^{1,1}(t)=E(t)\left(\Omega^{1,1}+\int_{0}^{t} E(s)^{-1} \hat{\psi}^{1,1}(z(s))\left(E(s)^{-1}\right)^{*} d s\right) E(t)^{*} .
$$


For large $n$, under the conditions of Theorem 4.12 and Corollary 5.2 , the distribution of $\sqrt{n}\left(Z^{n, 1,1}(t)-z^{1,1}(t)\right)$ can be approximated by the Normal distribution $\mathcal{N}\left(0, \Sigma^{1,1}(t)\right)$. In particular, we have

$$
\begin{aligned}
& \left(\operatorname{Var} Z_{X_{u}}(t), \operatorname{Var} Z_{X_{r}}(t), \operatorname{Var} Z_{X_{e}}(t), \operatorname{Var} N_{Y_{u}}(t), \operatorname{Var} N_{Y_{e}}(t)\right) \\
& \quad \simeq n\left(\operatorname{Var}\left(\eta_{X_{u}}(t)\right), \operatorname{Var}\left(\eta_{X_{r}}(t)\right), \operatorname{Var}\left(\eta_{X_{e}}(t)\right), \operatorname{Var}\left(\eta_{Y_{u}}^{1}(t)\right), \operatorname{Var}\left(\eta_{Y_{e}}^{1}(t)\right)\right) .
\end{aligned}
$$

This shows that variability in the numbers of individuals in the compartments is of order $\sqrt{n}$, explaining why simulations of the stochastic process in Temime et al (2003) show high variability.

If $\lambda_{e}$ or $\beta_{e}$ are not constant, but $P_{u}$ and $P_{e}$ are absolutely continuous with respect to Lebesgue measure, then $\left(x_{u}(t), x_{r}(t), x_{e}(t), \pi_{u}(t, m), \pi_{e}(t, m)\right)$ can be computed via numerical integration of (3.4). Monte Carlo simulations of $Z^{f n, f, g}(t)-z^{f, g}(t)$ can thus be performed for any measurable and bounded functions $f$ and $g$ in order to evaluate quantities of interest, such as times of emergence of resistant bacteria.

It is well known that the existence of a central limit theorem with Gaussian limit is central to the validity of bootstrap methods (Beran and Ducharme, 1991). In a situation where model parameters (or at least some of them) are estimated from observed data (e.g. by conditional least-squares), Theorem 4.12 may thus be invoked to establish the consistency of bootstrap methods.

\section{Discussion}

The issue of bacterial resistance to antibiotics is critical from a Public Health perspective. Since the emergence of resistant strains and their dissemination throughout the population results from the complex interaction of several processes, it is natural to rely on mathematical modeling to investigate the possible time course evolutions and to evaluate the impact of potential interventions.

Realistic descriptions of biological and epidemiological processes are often conveniently achieved through individual based models involving chance events. Numerical simulation of the resulting stochastic models using standard simulation algorithms may quickly raise feasibility issues due to computational load as the population size is increased and the structure of the compartments is detailed. The large number of simulations required to fully explore the model variability makes the problem even worse. For such reasons, a common practice is to fall back on a deterministic model in place of the initial stochastic formulation. Here, we showed that using the formalism of measure valued processes, it it possible to fully justify the choice of a deterministic formulation as the limiting case of the initial stochastic model. This is clearly an improvement on heuristic arguments as in (Temime et al 2005).

The law of large numbers provides a firm theoretical foundation for the deterministic model in the limit of a large population. Under assumptions which

allow for numerical evaluation of the deterministic limit, this limit provides a simple means for analyzing the temporal trends of bacterial antibioresistance. 
In this respect, the law of large numbers provides a theoretical confirmation the validity of the long term trends described in Temime et al (2005), such as convergence to a steady-state of the numbers of individuals in each compartment, together with an increase of the level of antibiotic resistance in carriers. This shows in particular that exposure to antibiotics is critical, and that the level of resistance will be increasing with time in the absence of intervention on this factor.

The central limit theorem specifies the rate of convergence and provides an explanation for the high variability of e.g. times to emergence of resistant bacterial strains described by Temime et al (2003), since it proves that for large $n$, the standard deviations of the process components are of order $\sqrt{n}$.

Regarding the particular model and situation studied in the present work, we note that the deterministic model used in (Temime et al, 2005) provided good qualitative agreement with the time trends of antibiotic resistance in the field (Temime et al, 2003). Including age structure in the model was worth considering, since the frequency of treatment by antibiotics, and the probability of colonization, depend on age, and since the analysis of a model with age structure raises no novel methodological issue. However, the model of Temime et al (2003) used an effective treatment frequency that was calculated by weighting observed frequencies of treatment with probabilities of colonization according to age.

Here, for the sake of definiteness, the initial numbers of individuals in the five compartments of the model were assumed to be multinomial. However, other distributions could be assumed instead. The proof of the law of large numbers carries over to any context in which Lemma 3.2 is valid, i.e. $Z^{n}(0)$ tends to a deterministic vector $z(0)$ as $n$ grows large. In particular, a deterministic initial configuration is suitable. If $Z^{n}(0)$ tends to a random limit, the convergence in Theorem 3.3 is in law rather than in probability.

The SIS model is a standard choice to study endemic diseases Andersson and Britton, 2000, chapter 8). Alternatively one may use SIR models with demography. Technically, the issue of an open population of unbounded size is dealt with by using localizing stopping times, such as in Clémençon et al (2008) for instance. This option would yield similar results, but at the price of greater variability in the central limit theorem due to superimposed fluctuations in population size.

\section{Acknowledgements}

We wish to thank Michèle Thieullen for helpful discussions, and Viet Chi Tran for clarification of several points.

\section{References}

Aldous D (1978) Stopping times and tightness. Ann Probability 6(2):335-340 
Andersson H, Britton T (2000) Stochastic epidemic models and their statistical analysis, Lecture Notes in Statistics, vol 151. Springer-Verlag, New York

Badrikian A (1996) Martingales hilbertiennes. Ann Math Blaise Pascal (S3):115-171

Beran R, Ducharme GR (1991) Asymptotic theory for bootstrap methods in statistics. Université de Montréal, Centre de Recherches Mathématiques, Montreal, QC

Cars O, Hogberg LD, Murray M, Nordberg O, Sivaraman S, Lundborg CS, So $\mathrm{AD}$, Tomson G (2008) Meeting the challenge of antibiotic resistance. British Medical Journal 337(7672):726-728

Clémençon S, Tran VC, de Arazoza H (2008) A stochastic SIR model with contact-tracing: large population limits and statistical inference. J Biol Dyn 2(4):392-414

EARS-Network (2012) Antimicrobial resistance surveillance in Europe 2011. Stockholm: ECDC

Ethier SN, Kurtz TG (2005) Markov processes. Characterization and convergence. Hoboken, NJ: John Wiley \& Sons

Ferland R, Fernique X, Giroux G (1992) Compactness of the fluctuations associated with some generalized nonlinear Boltzmann equations. Canad J Math 44(6):1192-1205

Fournier N, Méléard S (2004) A microscopic probabilistic description of a locally regulated population and macroscopic approximations. Ann Appl Probab 14(4):1880-1919

Jacod J, Shiryaev AN (2003) Limit theorems for stochastic processes, Grundlehren der Mathematischen Wissenschaften [Fundamental Principles of Mathematical Sciences], vol 288, 2nd edn. Springer-Verlag, Berlin

Joffe A, Métivier M (1986) Weak convergence of sequences of semimartingales with applications to multitype branching processes. Adv in Appl Probab 18(1):20-65

Kufner A, Opic B (1984) How to define reasonably weighted Sobolev spaces. Comment Math Univ Carolin 25(3):537-554

Kurtz TG (1981) Approximation of population processes, CBMS-NSF Regional Conference Series in Applied Mathematics, vol 36. Society for Industrial and Applied Mathematics (SIAM), Philadelphia, Pa.

Maher M, Alemyehu W, Lakew T, Gaynor B, Hang S, Cevallos V, Keenan J, Lietman T, Porco T (2012) The fitness cost of antibiotic resistance in streptococcus pneumoniae: insight from the field. PLoSOne 7(1):e29,407 
Martinez JL (2008) Antibiotics and antibiotic resistance genes in natural environments. Science 321(5887):365-367

Méléard S (1998) Convergence of the fluctuations for interacting diffusions with jumps associated with Boltzmann equations. Stochastics Stochastics Rep $63(3-4): 195-225$

Méléard S, Roelly S (1993) Sur les convergences étroite ou vague de processus à valeurs mesures. C R Acad Sci Paris Sér I Math 317(8):785-788

Méléard S, Tran VC (2012) Slow and fast scales for superprocess limits of agestructured populations. Stochastic Process Appl 122(1):250-276

Métivier M (1982) Semimartingales, de Gruyter Studies in Mathematics, vol 2. Walter de Gruyter \& Co., Berlin, a course on stochastic processes

Métivier M (1987) Weak convergence of measure valued processes using Sobolevimbedding techniques. In: Stochastic partial differential equations and applications (Trento, 1985), Lecture Notes in Math., vol 1236, Springer, Berlin, pp $172-183$

Oelschläger K (1990) Limit theorems for age-structured populations. Ann Probab 18(1):290-318

Pollard D (1984) Convergence of stochastic processes. Springer Series in Statistics, Springer-Verlag, New York

Prellner K, Hermansson A, White P, Melhus A, Briles D (1999) Immunization and protection in pneumococcal otitis media studied in a rat model. Microb Drug Resist 5(1):73-82

Rebolledo R (1980) Central limit theorems for local martingales. Z Wahrsch Verw Gebiete 51(3):269-286

Revuz D, Yor M (1991) Continuous martingales and Brownian motion, Grundlehren der Mathematischen Wissenschaften [Fundamental Principles of Mathematical Sciences], vol 293. Springer-Verlag, Berlin

Roelly-Coppoletta S (1986) A criterion of convergence of measure-valued processes: application to measure branching processes. Stochastics 17(1-2):43-65

Temime L, Boëlle PY, Thomas G (2005) Deterministic and stochastic modeling of pneumococcal resistance to penicillin. Math Popul Stud 12(1):1-16

Temime L, Boelle P, Courvalin P, Guillemot D (2003) Bacterial resistance to penicillin $\mathrm{G}$ by decreased affinity of penicillin-binding proteins: A mathematical model. EMERGING INFECTIOUS DISEASES 9(4):411-417 